\title{
THE MINAKSHISUNDARAM-PLEIJEL COEFFICIENTS FOR THE VECTOR VALUED HEAT KERNEL ON COMPACT LOCALLY SYMMETRIC SPACES OF NEGATIVE CURVATURE BY
}

ROBERTO J. MIATELLO ${ }^{1}$

\begin{abstract}
We use harmonic analysis on semisimple Lie groups to determine the Minakshisundaram-Pleijel asymptotic expansion for the trace of the heat kernel on natural vector bundles over compact, locally symmetric spaces of strictly negative curvature.
\end{abstract}

Introduction. Let $G$ be a connected, real semisimple Lie group of rank one with finite center. Let $G=K \cdot A \cdot N$ be an Iwasawa decomposition of $G$ and let $M$ be the centralizer of $A$ in $K$. Denote by $\mathfrak{g}=\mathfrak{l} \oplus \mathfrak{a} \oplus \mathfrak{n}$ the corresponding Iwasawa decomposition of $g$, the Lie algebra of $G$. We use the $G$-invariant Riemannian metric on $G / K$ induced by $a^{-1} \cdot B_{\mathrm{g}}\left(a^{-1}\right.$ is a convenient constant and $B_{\mathrm{g}}$ is the Killing form of $g)$. If $\left(\tau, V_{\tau}\right)$ is an irreducible representation of $K$ we form the homogeneous vector bundle $E_{\tau}=G \times{ }_{\tau} V_{\tau} \rightarrow G / K$.

There is unique $G$-invariant connection on $E_{\tau}$ such that if $s$ is a $C^{\infty}$ cross-section, $X \in \mathfrak{p}, \pi: G \rightarrow G / K$ is the canonical projection and $\pi_{*}$ designates the differential of $\pi$ at $e$ (the identity of $G$ ), then

$$
\nabla_{\pi_{*}(X)}(s)=\left.\frac{d}{d t}\right|_{t=0} s(\exp (t X) K)
$$

We denote by $\nabla^{2}$ the connection Laplacian on $\left(E_{\tau}, \nabla\right)$.

We consider now a discrete, torsion free subgroup $\Gamma$ of $G$ such that $\Gamma \backslash G$ is compact. We give to $X=\Gamma \backslash G / K$ the push down Riemannian metric. Then $X$ is the most general compact locally symmetric space of negative curvature. Also, $D=-\nabla^{2}$ pushes down to a nonnegative, elliptic differential operator on $\Gamma \backslash G$ $\times_{\tau} V_{\tau} \rightarrow X$. Let $\left\{\lambda_{n}\right\}=\operatorname{spec}(D)$ be the spectrum of $D$. As it is well known, $e^{-s D}$ exists and is trace-class for $s>0$. Moreover $\phi_{\tau}(s)=\operatorname{tr}\left(e^{-s D}\right)=\Sigma_{n} e^{-s \lambda_{n}}$ has an asymptotic expansion

$$
\phi_{\tau}(s) \sim s^{-d / 2}\left(\sum_{i=0}^{\infty} a_{i} s^{i}\right) \quad \text { as } s \downarrow 0
$$

$\left(c=\frac{1}{2} \operatorname{dim}(G / K)\right)$ and the coefficients $a_{i}$ are local Riemannian invariants of $X$ (see [ABP], [BGM], [MS] and [MP]). In this paper we will use harmonic analysis on $G$

Received by the editors May 10, 1978 and, in revised form, March 22, 1979.

AMS (MOS) subject classifications (1970). Primary 43A65, 58G99.

${ }^{1}$ The results of this article are part of the doctoral thesis of the author, written under the direction of Dr. N. R. Wallach at Rutgers University. The author wishes to thank Dr. Wallach for his help and encouragement. Thanks are also due to the referee for useful suggestions. 
to compute the coefficients $a_{i}$ of the above expansion. In essence our method is as follows.

Since $\Gamma$ is cocompact, the right regular representation $\pi_{\Gamma}$ of $G$ on $L^{2}(\Gamma \backslash G)$ decomposes

$$
\pi_{\Gamma}=\sum_{w \in \hat{G}} n_{\Gamma}(\omega) \cdot \omega
$$

and $n_{\Gamma}(\omega)<\infty$ for any $\omega \in \hat{G}$. Here $\hat{G}$ stands for the set of all equivalence classes of irreducible unitary representations of $G$. If $f$ is a $C^{\infty}$ function with compact support on $G$, the operator $\pi_{\Gamma}(f)=\int_{G} f(x) \cdot \pi_{\Gamma}(x) d x$ on $L^{2}(\Gamma \backslash G)$ is well defined and has a trace. Hence $\operatorname{tr} \pi_{\Gamma}(f)=\Sigma_{\omega} n_{\Gamma}(\omega) \cdot \theta_{\omega}(f)$, where $\theta_{\omega}$ denotes the character of the class $\omega$. On the other hand, by using the Selberg trace formula, we may compute $\operatorname{tr} \pi_{\Gamma}(f)$ in a different manner (see [Wa2, Theorem 6.7]). It has been proved by Wallach in [Wa3] that

$$
\phi_{\tau}(s)=\sum_{\omega} n_{\Gamma}(\omega) \cdot\left[\tau: \omega_{\mid K}\right] e^{s\left(\lambda_{\omega}-\lambda_{r}\right)}
$$

where $\left[\tau: \omega_{\mid K}\right]$ is the multiplicity of $\tau$ in the restriction of $\omega$ to $K$ and $\lambda_{\omega}, \lambda_{\tau}$ are respectively the eigenvalues of the Casimir elements of $G$ and $K$ when computed with respect to $a^{-1} \cdot B_{8}$. To study $\phi_{\tau}(s)$ near $s=0$ we wish to look at (1) as being the trace of $\pi_{\Gamma}\left(f_{\tau, s}\right)$ with $f_{\tau, s}$ a function to be determined and then to use the Selberg trace formula to estimate $\phi_{\tau}(s)$.

In the first place we notice that the trace formula applies to $K$-finite functions belonging to $\mathcal{C}^{l}(G)$ (see 2.14 and 2.16). Here if $p>0$, $e^{p}(G)$ denotes HarishChandra $p$-Schwartz space. What we need is therefore a $K$-finite function $f_{\tau, s} \in$ $\mathcal{C}^{1}(G)$ satisfying $\theta_{\omega}\left(f_{\tau, s}\right)=\left[\tau: \omega_{\mid K}\right] e^{s\left(\lambda_{\omega}-\lambda_{r}\right)}$ for all $\omega \in \hat{G}$. A candidate for $f_{\tau, s}$ is the continuous part of the heat kernel that we call $h_{\tau, s}$, defined by means of a wave packet in the sense of Harish-Chandra (see §4). Historically this function was introduced by Gangolli in the case $\tau=1$ to study $\phi_{1}(s)$ (see [Ga]). The main problem is the fact that unless $\tau$ and $G$ are very specific, $h_{\tau, s} \notin \mathcal{C}^{l}(G)$ (see remarks after Theorem 5.7). Hence $\pi_{\Gamma}\left(h_{\tau, s}\right)$ is in general not defined.

To avoid this problem we use a convenient decomposition of $h_{\tau, s}: h_{\tau, s}=h_{\tau, s}^{0}+$ $h_{\tau, s}^{1}$, where $h_{\tau, s}^{1} \in \mathcal{C}^{p}(G)$ for all $p>0$ and moreover $h_{\tau, s}^{1}$ has the same continuous Fourier transform as $h_{\tau, s}$. This decomposition is due to Campoli (see [C]). The Selberg trace formula is then applied, not to $h_{\tau, s}$ but to $h_{\tau, s}^{1}$, implying that

$$
\phi_{\tau}(s) \sim \operatorname{vol}(\Gamma \backslash G)\left(h_{\tau, s}(e)+\sum_{\omega \in \hat{G}_{d}} d(\omega)\left[\tau: \omega_{\mid K}\right] e^{s\left(\lambda_{\omega}-\lambda_{r}\right)}\right)
$$

where $\sim$ means that the difference between both sides tends to zero exponentially as $s \rightarrow 0$. Moreover, if $\omega \in \hat{G}_{d}, d(\omega)$ stands for the formal degree of $\omega$. This formula was proved by the author under the condition that $\tau \in \hat{G}$ restrict simply to $M$. The result for arbitrary $\tau$ is due to De George and Wallach (see [GWa]). Since the sum $\sum_{\omega \in \hat{G}_{d}} d(\omega)\left[\tau: \omega_{\mid K}\right] e^{s\left(\lambda_{\omega}-\lambda_{T}\right)}$ is computable (see [HS]), (2) implies that the asymptotic expansion of $\phi_{\tau}(s)$ as $s \rightarrow 0$ is determined by the expansion of $h_{\tau, s}(e)$ as $s \rightarrow 0$. We derive this expansion in $\S 6$. 
In [CGW] (see also [CW]) Cahn, Gilkey and Wolf obtain a proportionality principle that relates in a simple manner the expansion of $\phi_{\tau}(s)$ to the expansion of $\phi_{\tau}^{\prime}(s)$, the trace of the heat kernel on the vector bundle determined by $\tau$ over the compact dual $X^{\prime}$ of $X=\Gamma \backslash G / K$. In the light of this principle our formulas for the expansion of $\phi_{\tau}(s)$ imply similar formulas for the expansion of $\phi_{\tau}^{\prime}(s)$.

The outline of the exposition is as follows. In $\S 1$ we collect notation and recall some standard facts on homogeneous vector bundles over $G / K . \$ 2$ is devoted to the Selberg trace formula. We prove a sufficient condition on $f$, a $K$-finite function on $G$, for the trace formula to be valid for $f$ (see 2.14 and 2.16). $\$ 3$ contains the description of the problem. We next discuss some facts on representation theory necessary for the study of $h_{\tau, s}$. The main result of $\$ 4$ is Theorem 4.11 that establishes certain growth and functional properties of $h_{\tau, s^{\circ}}$ In $\$ 5$ we use results of De George to prove an asymptotic estimate (Theorem 5.1) and then use this estimate together with properties of $h_{\tau, s}$ to prove the main result (Theorem 5.7). \$6 is devoted to the computation of the asymptotic expansion of $h_{\tau, s}(e)$. To obtain it, we profit from the useful fact that as tempered distributions, $\tanh (\pi x)$ and $p \cdot v(\operatorname{coth} \pi x)$ have very simple Fourier transforms: $-i \cdot p v(\operatorname{cosech}(t / 2))$ and $-i$. $p v(\operatorname{coth}(t / 2))$ respectively.

1. Homogeneous vector bundles. In this section we follow closely the notation of Wallach [Wa1, Chapters 5 and 7]. Let $G$ be a connected semisimple Lie group with finite center. Let $G=K \cdot A \cdot N$ be an Iwasawa decomposition of $G$ and $\mathfrak{g}=\mathfrak{f} \oplus \mathfrak{a}$ $\bigoplus \mathfrak{n}$ be the corresponding decomposition at the algebra level. We will assume that $G$ has split rank one, that is, $\operatorname{dim} a=1$. Then, there is a real linear functional on $a$, we call it $\lambda$, such that

$$
\mathfrak{n}=\mathfrak{g}_{\lambda / 2} \oplus \mathfrak{g}_{\lambda}
$$

with $\mathfrak{g}_{j \cdot \lambda}=\{X \in \mathfrak{g} \mid[H, X]=j \cdot \lambda(H) \cdot X$, for any $H \in \mathfrak{a}\}\left(j=\frac{1}{2}, 1\right)$. The spaces $g_{\lambda / 2}$ and $g_{\lambda}$ are the restricted root spaces of $a$, corresponding to $\lambda / 2$ and $\lambda$, which are the positive restricted roots of a. Let $m(\lambda / 2)=\operatorname{dim}_{R} g_{\lambda / 2}, m_{\lambda}=\operatorname{dim}_{R} g_{\lambda}$ and $\rho=\frac{1}{2}\left(\frac{1}{2} m(\lambda / 2)+m(\lambda)\right) \lambda$. We will parametrize $a^{*}$ by $\lambda$, so that always $m(\lambda)$ is nonzero.

Choose $H_{1} \in$ a so that $\lambda\left(H_{1}\right)=2$. Any $a \in A$ can be written $a=a_{t}=\exp \left(t H_{1}\right)$, for a unique $t \in \mathbf{R}$. Set $A^{+}=\left\{a_{t} \mid t>0\right\}$. Let $B_{\mathfrak{g}}$ denote the Killing form of $\mathfrak{g}$, that is,

$$
B_{\mathrm{g}}(X, Y)=\operatorname{tr}(\operatorname{ad} X \cdot \operatorname{ad} Y) \text { for } X, Y \in \mathfrak{g} .
$$

The restriction of $B_{\mathrm{g}}$ to $a \times$ a puts in duality a with itself. Given $\mu \in \mathfrak{a}^{*}$ there is a unique element $H_{\mu} \in$ a so that $\mu(Z)=B_{\mathrm{g}}\left(H_{\mu}, Z\right)$ for all $Z \in \mathfrak{a}$.

We define an inner product on a by

$$
\left\langle Z_{1}, Z_{2}\right\rangle=\frac{B_{\mathrm{g}}\left(Z_{1}, Z_{2}\right)}{B_{\mathrm{g}}\left(H_{1}, H_{1}\right)} .
$$

On $a^{*}$ we use the dual inner product, that we also denote by $\langle$,$\rangle . With respect to$ these inner products,

$$
\left\|H_{1}\right\|^{2}=\left\langle H_{1}, H_{1}\right\rangle=1, \quad\|\lambda\|^{2}=\langle\lambda, \lambda\rangle=4
$$


and, if $b=$ number of different positive restricted roots of $a$, then

$$
\|\rho\|^{2}=\frac{1}{b^{2}}(m(\lambda / 2)+2 m(\lambda))^{2} .
$$

Let $\hat{K}$ denote the set of all equivalence classes of irreducible unitary representations of $K$. If $\left(\tau, V_{\tau}\right) \in \hat{K}$ set $d_{\tau}=\operatorname{dim}\left(V_{\tau}\right)$ and $\chi_{\tau}(k)=\operatorname{tr} \tau(k)$ for $\tau \in \hat{K}$. If $\sigma$ is an arbitrary representation of $K$ and $\tau \in \hat{K}$ we let $[\tau: \sigma]=\operatorname{dim} \operatorname{Hom}_{K}\left(V_{\tau}, V_{\sigma}\right)$.

For fixed $\sigma \in \hat{K}$, we form the $G$-vector bundle over $G / K$

$$
E=G \times{ }_{\sigma} V_{\sigma} \rightarrow M=G / K
$$

where $G \times{ }_{\sigma} V_{\sigma}=G \times V_{\sigma} / K$ and $K$ acts on the right on $G \times V_{\sigma}$ as usual: $(g, v) k=\left(g k, \sigma\left(k^{-1}\right) v\right), g \in G, v \in V, k \in K$. We write $[g, v]=(g, v) \cdot K$. Moreover, we define the left action of $G$ on $E$ by $x \cdot[g, v]=[x g, v]$.

If $\langle$,$\rangle is a K$-invariant inner product on $V_{o}$, then $\left\langle[g, v],\left[g, v^{\prime}\right]\right\rangle=\left\langle v, v^{\prime}\right\rangle$ is a well-defined unitary structure on $E$ and $G$ acts unitarily on the left in the obvious manner.

We now fix normalizations of Haar measures. Let $\mathfrak{p}$ be the orthogonal of with respect to $B_{\mathfrak{g}}$ and let $\theta: \mathfrak{g} \rightarrow \mathfrak{g}$ be the Cartan involution. Then

$$
\tilde{B}(X, Y)=-B(X, \theta Y)
$$

is an inner product on $\mathrm{g}$. We set $\langle\rangle=,\left(1 / B\left(H_{1}, H_{1}\right)\right) \cdot \tilde{B}$ (where $H_{1} \in \mathfrak{a}$ is such that $\lambda\left(H_{1}\right)=2$ and $\lambda$ is the long restricted root). Let $d \tilde{x}, d \tilde{k}$ and $d \bar{x}$ denote the Riemannian measures on $G, K$ and $G / K$ respectively, corresponding to the Riemannian structures induced by $\langle$,$\rangle in the standard fashion. If \operatorname{vol}(K)$ is the volume of $K$ relative to $d \tilde{k}$, let $d x=\operatorname{vol}(K)^{-1} \cdot d \tilde{x}$ and $d k=\operatorname{vol}(K)^{-1} \cdot d \tilde{k}$. With respect to these normalizations

for any $f \in C_{c}(G)$.

$$
\int_{G / K} \int_{K} f(x k) d k d \bar{x}=\int_{G} f(x) d x
$$

Let $C^{\infty}(E)$ and $C_{c}^{\infty}(E)$ be respectively the set of $C^{\infty}$ cross-sections of $E$ and of $C^{\infty}$, compactly supported cross-sections of $E$. If $1<p<\infty$ let $L^{p}(E)$ be the set of $p$-integrable cross-sections of $E$. We now recall the standard correspondence between cross-sections of $E=G \times_{\sigma} V_{\sigma}$ and certain $V_{\sigma}$-valued functions on $G$. Set

$$
\begin{aligned}
C^{\infty}(G: \sigma)=\left\{h \mid h: G \rightarrow V_{\sigma},\right. & h \text { is } C^{\infty} \\
& \text { and } \left.h(g k)=\sigma(k)^{-1} h(g) \text { for } g \in G, k \in K\right\} .
\end{aligned}
$$

Let $A: C^{\infty}(E) \rightarrow C^{\infty}(G: \sigma)$ be so that $A f(g)=g^{-1} f(g K)$, for $f \in C^{\infty}(E)$. Define, for $h \in C^{\infty}(G: \sigma), B(h)(g K)=[g, h(g)]$. Then $B(h) \in C^{\infty}(E)$ and $(A \cdot B)(h)=h$, $(B A)(f)=f$, for $h \in C^{\infty}(G: \sigma)$ and $f \in C^{\infty}(E)$. Moreover $A$ intertwines the actions of $G$, where $(g \cdot f)(x K)=g f\left(g^{-1} x K\right)$ and $(g h)(x)=h\left(g^{-1} x\right)$, for $f \in$ $C^{\infty}(E), h \in C^{\infty}(G: \sigma)$. Finally $A$ preserves the $p$-norms.

Let now $D$ be a $G$-invariant differential operator on $E$. The action of $G$ on $C^{\infty}(E)$ induces an action of $\mathcal{U}(g)$, the universal enveloping algebra of $g$, on $C^{\infty}(E)$ so that if $f \in C^{\infty}(E)$ and $X \in \mathfrak{g}$,

$$
(X f)(g K)=\left.\frac{d}{d t}\right|_{t=0} \exp (t X) \cdot f(\exp (-t X) g K) .
$$


If $X_{1}, X_{2}, \ldots, X_{n}$ is a basis of $g$, let $Y_{1}, Y_{2}, \ldots, Y_{n}$ be the dual basis with respect to $\langle\rangle=,\left(1 / B_{\mathrm{g}}\left(H_{1}, H_{1}\right)\right) \cdot B_{\mathrm{g}}$.

Put $\Omega=\sum_{1}^{n} X_{i} \cdot Y_{i} \in Q(\mathrm{~g})$. Then $\Omega$ is clearly independent of the choice of basis and in particular, $\operatorname{Ad}(g) \Omega=\Omega$. Thus $\Omega$ defines a $G$-invariant elliptic differential operator on $E$ that we denote $\Omega_{E}$.

We define a $G$-invariant connection on $E$ by putting

$$
\nabla_{g_{*}} f=\left.\frac{d}{d t}\right|_{t=0} g(g \exp (t X))^{-1} f(g \exp (t X) K),
$$

where $f \in C^{\infty}(E), v \in T(G / K)_{e K}$ and $X \in \mathfrak{p}$ is unique with the property that $v=\pi_{*}(X)$.

1.1 Proposition. Let $\nabla^{2}$ denote the connection Laplacian on $E$. Then there is a scalar $\lambda_{\sigma}$ so that $\Omega=\nabla^{2}+\lambda_{\sigma} \cdot I$.

Proor. Let for $h \in C^{\infty}(G: \sigma)$ and $X \in g$

$$
\left(R_{X} h\right)(g)=\left.\frac{d}{d t}\right|_{t=0} h(g \cdot \exp t X) .
$$

Then if $f \in C^{\infty}(E)$ and $X_{1}, X_{2}, \ldots, X_{d}$ is an orthonormal basis of $\mathfrak{p}$ with respect to $\langle$,$\rangle ,$

$$
A\left(\nabla^{2} f\right)=\sum_{i=1}^{d} R_{X_{i}}^{2}(A f)
$$

A computation shows that if $X \in \mathfrak{g}, f \in C^{\infty}(E)$ then

$$
A\left(X^{2} f\right)(g)=\left(R_{-\operatorname{Ad}\left(g^{-1}\right)(X)}^{2}(A f)\right)(g) .
$$

Hence, if $X_{1}, X_{2}, \ldots, X_{d}$ is a basis of $\mathfrak{p}$ and $Y_{1}, Y_{2}, \ldots, Y_{m}$ is a basis of $f$ such that $\left\langle X_{i}, X_{j}\right\rangle=\delta_{i j},\left\langle Y_{i}, Y_{j}\right\rangle=-\delta_{i j}$ then $A(\Omega f)=A\left(\nabla^{2} f\right)-\Sigma_{j} R_{Y}^{2}(A f)$.

Now $\Omega_{K}=-\Sigma_{j} Y_{j}^{2}$ lies in the center of $U(\mathfrak{f})$, the universal enveloping algebra of $\mathfrak{f}$; hence if $\sigma \in \hat{K}$, then $\sigma\left(\Omega_{K}\right)=\lambda_{\sigma} \cdot I$ for some $\lambda_{\sigma} \in \mathbf{C}$. It is easy to check that $-\Sigma_{j} R_{Y}^{2}(A f)=\lambda_{\sigma} A(f)$. Q.E.D.

Let now $\Gamma$ be a discrete torsion free subgroup of $G$ such that $\Gamma \backslash G$ is compact. This assumption says that $\Gamma \backslash G / K$ is a manifold since $\Gamma$ acts freely and properly discontinuously on $G / K$. Since $\Gamma$ acts by isometries, we may push down the Riemannian structure of $G / K$ to $\Gamma \backslash G / K$ so that $\pi: G / K \rightarrow \Gamma \backslash G / K$ is a local isometry. The operators $\Omega_{E}$ and $\nabla^{2}$ push down to elliptic operators on $\Gamma \backslash E$; we call them $\Omega_{\Gamma}$ and $\nabla_{\Gamma}^{2}$ and we still have $\Omega_{\Gamma}=\nabla_{\Gamma}^{2}+\lambda_{\sigma} \cdot I$. Finally, since the action of $\Gamma$ on $E$ is unitary $\Gamma \backslash E$ has a well defined unitary structure. We fix a measure $d \dot{g}$ on $\Gamma \backslash G$ so that

$$
\int_{\Gamma \backslash G}\left(\sum_{\gamma} f(\gamma x)\right) d \dot{x}=\int_{G} f(x) d x, \text { for } f \in C_{c}(G) .
$$

On $\Gamma \backslash G / K$ we use the Riemannian measure $d \bar{x}$. With respect to these measures,

$$
\int_{\Gamma \backslash G / K} \int_{K} h(x k) d k d \bar{x}=\int_{\Gamma \backslash G} h(x) d \dot{x}, \quad \text { for } h \in C(\Gamma \backslash G) .
$$


If $1 \leqslant p<\infty$ let $\|f\|_{p}=\int_{\Gamma \backslash G / K}|f(x)|^{p} d x$ and $L^{p}(\Gamma \backslash E)=\{f \mid f=$ cross-section of $\Gamma \backslash E \rightarrow \Gamma \backslash M$ st $\left.\||f|\|_{p}<\infty\right\}$.

We still have a correspondence between cross-sections and vector-valued functions on the group.

1.2 Lemma. Let $C^{\infty}(\Gamma \backslash G: \sigma)=\left\{f: G \rightarrow V_{o} \mid f\right.$ is $C^{\infty}$ and $f(\gamma g k)=\sigma\left(k^{-1}\right) f(g)$ for $\gamma \in \Gamma, g \in G, k \in K\}$.

Let $B_{\Gamma}: C^{\infty}(\Gamma \backslash G: \sigma) \rightarrow C^{\infty}(\Gamma \backslash E)$ be $B_{\Gamma}(f)(\Gamma x K)=\Gamma \cdot B f(x K)$ where $B$ : $C^{\infty}(G: \sigma) \rightarrow C^{\infty}(E)$ satisfies $B(h)(g K)=[g, h(g)]$. Then $B_{\Gamma}$ is an isomorphism and preserves the p-norms.

Proof. If $f \in C^{\infty}(E)$ satisfies $f(\gamma x)=\gamma f(x)$, for $\gamma \in \Gamma, x \in G / K$ set $\tilde{f}(\Gamma x)=\Gamma$ - $f(x)$. Then $\tilde{f} \in C^{\infty}(\Gamma \backslash E)$ and if $f_{1} \in C^{\infty}(\Gamma \backslash E)$ there is a unique $f \in C^{\infty}(E)$ so that $f(\gamma x)=\gamma f(x)$ and $\tilde{f}=f_{1}$. Indeed, since the action of $\Gamma$ on $M$ is free, if $[v]=f_{1}(\Gamma x)$ there is a unique $v \in E_{x}$, the fiber of $E$ at $x$, so that $\pi(v)=[v]$ where $\pi: E \rightarrow \Gamma \backslash E$ is the canonical projection. This globally defines the desired $f$. The first part of the lemma follows, since the $\Gamma$-equivariant cross-sections of $E$ correspond bijectively with the $\Gamma$-invariant elements of $C^{\infty}(G: \sigma)$. The $p$-norms are preserved since

$$
\int_{\Gamma \backslash G / K}\left\langle(B f)^{\sim}(\dot{x}),(B f)^{\sim}(\dot{x})\right\rangle_{\Gamma x K}^{p / 2} d \dot{x}=\int_{\Gamma \backslash G}\langle f(\dot{x}), f(\dot{x})\rangle^{p / 2} d \dot{x} . \quad \text { Q.E.D. }
$$

2. The Selberg trace formula. We keep the notation of $\S 1$. Let $\pi_{\Gamma}$ denote the right regular representation of $G$ on $L^{2}(\Gamma \backslash G)$. It is a well-known fact that $\pi_{\Gamma}$ decomposes $\pi_{\Gamma}=\sum_{\omega \in \hat{G}} n_{\Gamma}(\omega) \cdot \omega$ and $n_{\Gamma}(\omega)<\infty$ for $\omega \in \hat{G}$, where as usual, $\hat{G}$ stands for the set of all equivalence classes of irreducible unitary representations of $G$. We now briefly describe the trace formula on $L^{2}(\Gamma \backslash G)$. Our source is Wallach [Wa2].

If $\phi \in C_{c}^{\infty}(G), \omega \in \hat{G}$ and $\left(\pi_{\omega}, H_{\omega}\right) \in \omega$, the operator $\pi_{\omega}(\phi)=\int_{G} \phi(x) \cdot \pi_{\omega}(x) d x$ on $H_{\omega}$ is trace class and its trace $\theta_{\omega}(\phi)=\operatorname{tr} \pi_{\omega}(\phi)$ (the character of $\omega$ ) completely characterizes the class $\omega$.

The operator on $L^{2}(\Gamma \backslash G), \pi_{\Gamma}(\phi)=\int_{G} \phi(x) \cdot \pi_{\Gamma}(x) d x$ is a kernel operator with $C^{\infty}$ kernel $k(x, y)=\Sigma_{\gamma \in \Gamma} \phi\left(y^{-1} \cdot \gamma \cdot x\right)$, the series converging uniformly on compacta. Because $k(x, y)$ is $C^{\infty}, \pi_{\Gamma}(\phi)$ is of trace class and

$$
\operatorname{tr} \pi_{\Gamma}(\phi)=\int_{\Gamma \backslash G} k(x, x) d \dot{x}=\int_{\Gamma \backslash G} \sum_{\gamma} \phi\left(x^{-1} \gamma x\right) d \dot{x} .
$$

Set for $\gamma \in \Gamma$

$[\gamma]=$ the $\Gamma$-conjugacy class of $\gamma$.

$[\Gamma]=$ the set of all $\Gamma$-conjugacy classes of elements of $\Gamma$.

$G_{\gamma}=$ the centralizer of $\gamma$ in $G$.

$\Gamma_{\gamma}=\Gamma \cap G_{\gamma}$.

Also we normalize the measures on $G_{\gamma}$ and $G_{\gamma} \backslash G$ so that for $\psi, \eta \in C_{c}(G)$

$$
\begin{aligned}
& \int_{G} \psi(g) d g=\int_{G_{\gamma} \backslash G} \int_{G_{\gamma}} \psi(x g) d x d \dot{g}, \\
& \int_{G_{\gamma}} \eta(g) d g=\int_{\Gamma_{\gamma} \backslash G_{\gamma}} \sum_{\tau \in \Gamma_{\gamma}} \eta(\tau g) d \dot{g} .
\end{aligned}
$$


With these normalizations we have

$$
\begin{aligned}
\operatorname{tr} \pi_{\Gamma}(\phi) & =\sum_{\omega \in \hat{G}} n_{\Gamma}(\omega) \cdot \theta_{\omega}(\phi) \\
& =\sum_{[\gamma] \in[\Gamma]} \operatorname{vol}\left(\Gamma_{\gamma} \backslash G_{\gamma}\right) \int_{G_{\gamma} \backslash G} \phi\left(g^{-1} \gamma g\right) d g .
\end{aligned}
$$

As in $\S 1$, let $H_{1} \in$ a satisfy $\lambda\left(H_{1}\right)=2$. Then $A=\left\{a_{t}=\exp \left(t H_{1}\right) \mid t \in \mathbf{R}\right\}$.

Since all elements $\gamma \in \Gamma$ are semisimple (see e.g. Raghunathan [R]) and $\Gamma$ is torsion free, it follows that for any $\gamma \in \Gamma$ there exists $g \in G$ so that

$$
g^{-1} \gamma g=m_{\gamma} \cdot \exp \left(t_{\gamma} \cdot H_{1}\right) \quad\left(m_{\gamma} \in M, t_{\gamma}>0\right) .
$$

Here $t_{\gamma}$ happens to be independent of the choice of $g$ or the particular Iwasawa decomposition and so is $m_{\gamma}$, up to conjugacy in $M$. Therefore, the following are well defined:

$$
\begin{aligned}
D(\gamma) & =D\left(m_{\gamma} \cdot \exp \left(t_{\gamma} H_{1}\right)\right) \\
& =\exp \left(-t_{\gamma}\|\rho\|\right) \cdot\left|\operatorname{det}\left(\operatorname{Ad}\left(m_{\gamma} \cdot \exp \left(t_{\gamma} H_{1}\right)\right)^{-1}-\left.I\right|_{\mathfrak{n}}\right)\right|, \\
u(\gamma) & =\operatorname{vol}\left(A \backslash G_{m_{\gamma} \cdot \exp \left(t_{\gamma} H_{1}\right)}\right) \text { and } \chi_{\sigma}\left(m_{\gamma}\right)=\operatorname{tr} \sigma\left(m_{\gamma}\right) .
\end{aligned}
$$

Here $b=$ number of distinct positive restricted roots (i.e, $b=1$ if $m(\lambda / 2)=0$ and $b=2$ if $m(\lambda / 2) \neq 0$ ).

Finally, we let for $\sigma \in \hat{M}$ and $\nu \in a_{\mathbf{C}}^{*}, \pi_{\sigma, \nu}$ be the principal series representations of $G$, that is, $\pi_{\sigma, \nu} \simeq \tilde{\pi}^{\sigma, \rho+i \nu}$ in the notation of [Wa1, Chapter 8].

2.1 Theorem.(See [W, Theorem 6.7].) If $\phi \in C_{c}^{\infty}(G)$ then

$$
\begin{aligned}
\operatorname{tr} \pi_{\Gamma}(\phi)= & \sum_{\omega \in \hat{G}} n_{\Gamma}(\omega) \cdot \theta_{\omega}(\phi) \\
= & \operatorname{vol}(\Gamma \backslash G) \cdot \phi(e)+\frac{1}{2 \pi} \sum_{[\gamma] \in[\Gamma]-[e]} \operatorname{vol}\left(\Gamma_{\gamma} \backslash G_{\gamma}\right) D(\gamma)^{-1} \\
& \cdot u(\gamma)^{-1} \sum_{\sigma \in \hat{M}} \overline{\chi_{0}\left(m_{\gamma}\right) \cdot b} \int_{-\infty}^{+\infty} \theta_{\pi_{\sigma, \nu \lambda}}(\phi) e^{-2 i i_{\gamma} \nu} d \nu .
\end{aligned}
$$

If $\phi \in L^{1}(G)$ instead of $\phi \in C_{c}^{\infty}(G)$, the operator $\pi_{\Gamma}(\phi)$ is still defined but it need not be true that $\pi_{\Gamma}(\phi)$ is trace class. The objective of the rest of the section is to give a sufficient condition for $\phi \in L^{1}(G)$ to be put into the trace formula. We observe that all we need for the validity of the trace formula for $\phi$ is that the series $\Sigma_{\gamma \in \Gamma} \phi\left(y^{-1} \gamma x\right)$ converges uniformly on compacta to a sufficiently regular kernel, because then the operator $\pi_{\Gamma}(\phi)$ has to be given by this kernel and will be trace class because of the regularity of the kernel. Throughout the rest of this section we may drop the assumption that $G$ be of split rank one.

2.2 LEMMA. Let $M$ be a compact manifold of dimension $n$ with volume form $\omega$. Let $k: M \times M \rightarrow \mathrm{C}$ be of class $C^{(n+1)}$. Then the operator

$$
(K f)(x)=\int_{M} k(x, y) \cdot f(y) d \omega(y) \quad\left(f \in L^{2}(M, \omega)\right)
$$

is trace class and $\operatorname{tr} K=\int_{M} k(x, x) d \omega(x)$. 
Proof. For $M=T^{n}$ the theorem follows from the fact that if

$$
k(x, y)=\sum_{\alpha, \beta \in \mathbf{Z}^{n}} a_{\alpha, \beta} e^{i \alpha \cdot x} \cdot e^{-i \beta y}
$$

and $k(x, y) \in C^{(n+1)}\left(T^{2 n}\right)$ then $\Sigma_{\alpha, \beta}\left|a_{\alpha \beta}\right|<\infty$. For a general compact manifold, the obvious partition of unity argument reduces the problem to $M=T^{n}$.

2.3 THEOREM. Let $\left(\tau, V_{\tau}\right)$ be a unitary representation of $K, r$ a natural number and $n=\operatorname{dim} G$. Assume that $f: G \rightarrow \operatorname{End}\left(V_{\tau}\right)$ is $C^{\infty}$ and $f\left(k_{1} g k_{2}\right)=\tau\left(k_{1}\right) \cdot f(g) \cdot \tau\left(k_{2}\right)$, for $k_{i} \in K, g \in G$ and that $\Omega^{j} f \in L^{1}$ for $0 \leqslant j<n+[r / 2]+1$. Then $\Sigma_{\gamma} f\left(g^{-1} \gamma x\right)$ converges almost everywhere to a $C^{r}$ function on $\Gamma \backslash G \times \Gamma \backslash G$.

Proof. Let $\left(\rho\right.$, End $\left.V_{\tau}\right)$ be the unitary representation of $K \times K$ with action and inner product given by $\rho\left(k_{1}, k_{2}\right) T=\tau\left(k_{1}\right) \cdot T \cdot \tau\left(k_{2}\right)^{-1},\langle T, U\rangle=\operatorname{tr}\left(U^{*} T\right)$. The series $\Sigma_{\gamma} f\left(g^{-1} \gamma x\right)$ converges almost everywhere to an integrable function $\phi(g, x)$ in $\Gamma \backslash G \times \Gamma \backslash G$, since

$$
\begin{aligned}
\int_{\Gamma \backslash G} \int_{\Gamma \backslash G} \sum_{\gamma \in \Gamma}\left\|f\left(g^{-1} \gamma x\right)\right\| d \dot{g} d \dot{x} & =\int_{\Gamma \backslash G} \int_{G}\left\|f\left(g^{-1} x\right)\right\| d g d \dot{x} \\
& =\|f\|_{1} \cdot \operatorname{vol}(\Gamma \backslash G) .
\end{aligned}
$$

Also, $\phi\left(g k_{1}, x k_{2}\right)=\rho\left(k_{1}, k_{2}\right)^{-1} \cdot \phi(g, x)$. Thus, in the notation of $\S 1$,

$$
\phi \in L^{1}(\Gamma \times \Gamma \backslash G \times G: \rho) \text {. }
$$

We will compute $\Omega_{\rho} \phi, \Omega_{\rho}$ being the Laplacian on the vector bundle

$$
\Gamma \times \Gamma \backslash G \times G \times{ }_{p} K \times K \rightarrow \Gamma \times \Gamma \backslash G \times G / K \times K
$$

Let $\psi \in C^{\infty}(\Gamma \times \Gamma \backslash G \times G: \rho)$. Then if $X \in \mathfrak{g}$,

$$
\begin{aligned}
\int_{\Gamma \backslash G} \int_{\Gamma \backslash G} \sum_{\gamma} f\left(g^{-1} \gamma x\right) \cdot X_{x} \psi(g, x) d \dot{x} d \dot{g} \\
\quad=\left.\int_{\Gamma \backslash G} \frac{d}{d t}\right|_{t=0} \int_{\Gamma \backslash G} \sum_{\gamma} f\left(g^{-1} \gamma x\right) \cdot \psi(g, x \exp (t X)) d \dot{x} d \dot{g}
\end{aligned}
$$

By dominated convergence the above equals

$$
\begin{array}{rl}
\left.\int_{\Gamma \backslash G} \int_{G} \frac{d}{d t}\right|_{t}=0 & f\left(g^{-1} x \exp (-t X)\right) \cdot \psi(g, x) d x d \dot{g} \\
& =\int_{\Gamma \backslash G} \int_{G}(-X f)\left(g^{-1} x\right) \psi(g, x) d x d \dot{g} \\
& =\int_{\Gamma \backslash G} \int_{\Gamma \backslash G}\left(\sum_{\gamma}(-X f)\left(g^{-1} \gamma x\right)\right) \psi(g, x) d \dot{x} d \dot{g}
\end{array}
$$

Therefore, for $j=1,2, \ldots, n+[r / 2]+1$, we have that in the sense of distributions

$$
\left(\Omega_{x}^{j} \phi\right)(g, x)=\sum_{\text {a.e. }} \sum_{\gamma} \Omega^{j} f\left(g^{-1} \gamma x\right) \in L^{1}(\Gamma \backslash G \times \Gamma \backslash G)
$$


since $\Omega^{j} f \in L^{1}(G)$, for $1<j<n+[r / 2]+1$. Similarly

$$
\begin{aligned}
\int_{\Gamma \backslash G} \int_{\Gamma \backslash G}\left(\sum_{\gamma} f\left(g^{-1} \gamma x\right)\right) X_{g} \psi(g, x) d \dot{g} d \dot{x} \\
=\int_{\Gamma \backslash G} \int_{G}\left(R_{\mathrm{Ad}\left(x^{-1} g\right)(-x)} f\right)\left(g^{-1} x\right) \cdot \psi(g, x) d g d \dot{x}
\end{aligned}
$$

This implies that

$$
\begin{aligned}
\int_{\Gamma \backslash G} \int_{\Gamma \backslash G} \phi(g, x) \Omega_{g} \psi(g, x) d \dot{g} d \dot{x} \\
=\int_{\Gamma \backslash G} \int_{\Gamma \backslash G}\left(\sum_{\gamma} \Omega f\left(g^{-1} \gamma x\right)\right) \cdot \psi(g, x) d \dot{g} d \dot{x}
\end{aligned}
$$

Thus

$$
\begin{aligned}
\int_{\Gamma \backslash G} \int_{\Gamma \backslash G} \phi(g, x) \Omega_{x} & \times \Omega_{g} \psi(g, x) d \dot{g} d \dot{x} \\
& =\int_{\Gamma \backslash G} \int_{\Gamma \backslash G}\left(\sum_{\gamma} 2 \Omega f\left(g^{-1} \gamma x\right)\right) \psi(g, x) d \dot{g} d \dot{x} .
\end{aligned}
$$

That is, as distributions, for any $j=1,2, \ldots, n+[r / 2]+1$,

$$
\left(\Omega_{\rho}^{j} \phi\right)(g, x)=\sum_{\gamma \in \Gamma} 2^{j} \Omega^{j} f\left(g^{-1} \gamma x\right) \in L^{1}(\Gamma \times \Gamma \backslash G \times G: \rho) .
$$

For $s \in \mathbf{R}$, let $H^{s}$ denote the $s$-Sobolev space of the vector bundle $\Gamma \times \Gamma \backslash G \times$ $G \times{ }_{p} K \times K \rightarrow \Gamma \times \Gamma \backslash G \times G \backslash K \times K$ (cf. Palais et al. [P]).

It now follows that

$$
\begin{aligned}
& \Omega_{\rho}^{n+[r / 2]+1}(\phi) \in L^{1}(\Gamma \times \Gamma \backslash G \times G: \rho) \\
& \simeq L^{1}\left(\Gamma \times \Gamma \backslash G \times G \times{ }_{\rho} K \times K\right) \subset H^{-n-e}, \text { for any } \varepsilon>0
\end{aligned}
$$

But $\Omega_{\rho}$ is elliptic of order 2 . Hence

$$
\phi \in H^{-n-\varepsilon+2(n+[r / 2]+1)} \subset H^{n+r+1-\varepsilon} .
$$

This completes the proof since $H^{n+r+\delta} \subset C^{r}$, for any $\delta>0$. Q.E.D.

We wish to prove a similar result for scalar valued functions. Let for $f \in C(G)$ and $h \in C(K)$

$$
\begin{aligned}
& (f * h)(x)=\int_{K} f(x k) h\left(k^{-1}\right) d k, \\
& (h * f)(x)=\int_{K} h(k) \cdot f\left(k^{-1} x\right) d k .
\end{aligned}
$$

A function $f \in C(G)$ is left $K$-finite (resp. right $K$-finite) if the left (resp. right) $K$-translates of $f:\left\{L_{k}(f) \mid k \in K\right\}$ (resp. $\left\{R_{k} f \mid k \in K\right\}$ ) span a finite dimensional subspace of $C(G)$.

2.4 Lemma. (a) $f \in C(G)$ is left $K$-finite if and only if there are $\tau_{1}, \tau_{2}, \ldots, \tau_{n} \in \hat{K}$ such that

$$
\sum_{i=1}^{n} d_{\tau_{i}} \overline{x_{r_{i}}} * f=f
$$


(b) $f \in C(G)$ is right $K$-finite if and only if there are $\tau_{1}, \tau_{2}, \ldots, \tau_{n} \in \hat{K}$ such that

$$
\sum d_{\tau_{i}} f * \chi_{\tau_{i}}=f
$$

2.5 LeMma. If $A \in \operatorname{End}\left(V_{\tau}\right)$ then

$$
d_{\tau} \int_{K} \operatorname{tr}\left(\tau\left(k^{-1}\right) A\right) \cdot \tau(k) d k=A
$$

Proof. Let $e_{1}, e_{2}, \ldots, e_{n}$ be an orthonormal basis of $V_{\tau}$. Then

$$
\begin{aligned}
\left\langle d_{\tau} \int_{K} \operatorname{tr}\left(\tau\left(k^{-1}\right) A\right) \cdot \tau(k) d k\left(e_{m}\right), e_{l}\right\rangle & \\
& =d_{\tau}\left\langle\sum_{i, j} \int\left\langle\tau\left(k^{-1}\right) e_{j}, e_{i}\right\rangle\left\langle A e_{i}, e_{j}\right\rangle \tau(k) d k\left(e_{m}\right), e_{l}\right\rangle \\
& =\left\langle A e_{m}, e_{l}\right\rangle \text {. Q.E.D. }
\end{aligned}
$$

2.6 LEMMA. Let

$$
\begin{gathered}
X_{\tau}=\left\{\phi: G \rightarrow \operatorname{End}\left(V_{\tau}\right) \mid \phi\right. \text { is continuous and } \\
\left.\quad \phi\left(k_{1} g k_{2}\right)=\tau\left(k_{1}\right) \phi(g) \tau\left(k_{2}\right), k_{i} \in K, g \in G\right\}, \\
Y_{\tau}=\left\{f: G \rightarrow C \mid f \text { is } K \text {-central and } d_{\tau} \chi_{\tau} * f=f\right\} .
\end{gathered}
$$

Set, for $\phi \in X_{\tau}, A(\phi)=\operatorname{tr} \phi$. Then $A: X_{\tau} \rightarrow Y_{\tau}$ is an isomorphism.

Proof. If $\left\{e_{i}\right\}_{1}^{n}$ is an orthonormal basis of $V_{\tau}$, then by Lemma 2.5

Thus $A(\phi) \in Y_{\tau}$, if $\phi \in X_{\tau}$. Now let

$$
\begin{aligned}
d_{\tau}\left(\chi_{\tau} * \operatorname{tr} \phi\right)(x) & =\int d_{\tau} \chi_{\tau}(k) \operatorname{tr} \phi\left(k^{-1} x\right) d k \\
& =\sum_{i}\left\langle\phi(x) e_{i}, e_{i}\right\rangle=\operatorname{tr} \phi(x) .
\end{aligned}
$$

$$
B(f)(g)=d_{\tau} \int_{K} f(g k) \cdot \tau\left(k^{-1}\right) d k, \quad f \in Y_{\tau} .
$$

It is easy to check that $B(f) \in X_{\tau}$ and that the maps $A$ and $B$ are inverses of each other. Q.E.D.

Lemmas 2.4 and 2.6 imply

2.7 Corollary. If $f \in C(G)$ is $K$-central and $K$-finite then $f=\sum_{i=1}^{n} \operatorname{tr}\left(\phi_{i}\right)$ for some $\phi_{i} \in X_{\tau_{i}}$ and $\tau_{i} \in \hat{K}, i=1,2, \ldots, n$.

2.8 REMARK. The following more general version of the corollary is true:

If $f \in C(G)$ is left and right $K$-finite then there exist $K$-types $\left(\tau_{i}, V_{\tau_{i}}\right),\left(\sigma_{i}, V_{\sigma_{i}}\right)$, vectors $v_{i} \in V_{\tau_{i}}, \omega_{i} \in V_{\sigma_{i}}$, continuous functions $\phi_{i}: G \rightarrow \operatorname{Hom}\left(V_{\tau_{i}}, V_{\sigma_{i}}\right)$ such that $\phi_{i}\left(k_{1} g k_{2}\right)=\sigma_{i}\left(k_{1}\right) \phi_{i}(g) \tau_{i}\left(k_{2}\right)$ and

$$
f(g)=\sum_{i=1}^{n}\left\langle\phi_{i}(g) v_{i}, w_{i}\right\rangle .
$$

2.9 COROLLARY. Let $r$ be a natural number. If $f$ is a $K$-central, $K$-finite function on $G$ such that $\Omega^{j} f \in L^{1}(G)$ for $j=1,2, \ldots, n+[r / 2]+1$, then $\Sigma_{\gamma} f\left(g^{-1} \gamma x\right)$ converges almost everywhere to a $C^{r}$ function on $\Gamma \backslash G \times \Gamma \backslash G$. 
Proof. This is a direct consequence of Theorem 2.3 and Corollary 2.7 since $f$ can be written $f=\sum \operatorname{tr} \phi_{i}$ and $\Omega^{j} f=\Sigma_{i} \operatorname{tr} \Omega^{j} \phi_{i} \in L^{1}(G)$ if and only if $\Omega^{j} \phi_{i} \in$ $L^{1}(\tau: G: \tau)$.

In particular if $f$ is as above with $r=n+1+[(n+1) / 2]$, by $2.3, \pi_{\Gamma}(f)$ is a kernel operator with kernel given by

$$
\phi(g, x) \underset{\text { a.e. }}{=} \sum f\left(g^{-1} \gamma x\right) \in C^{(n+1)}(\Gamma \backslash G \times \Gamma \backslash G) .
$$

By 2.2 we thus have,

2.10 Corollary. Let $f \in C^{3(n+1)}(G), f K$-central and $K$-finite. Assume also that $\Omega^{k} f \in L^{1}(G)$ for $k=1,2, \ldots,(n+1)+[(n+1) / 2]$. Then $\pi_{\Gamma}(f)$ is trace class on $L^{2}(\Gamma \backslash G)$ and $\operatorname{tr} \pi_{\Gamma}(f)=\int_{\Gamma \backslash G} \phi(x, x) d \dot{x}$.

At this point we observe that if the convergence of the series $\Sigma_{\gamma} f\left(g^{-1} \gamma x\right)$ were uniform on compacta, then the conditions of 2.10 would be enough to ensure that $f$ goes into the trace formula (Theorem 2.1). To fill this gap we will need to impose another mild condition on $f$.

2.11 Definition. A function $f \in C(G) \cap L^{1}(G)$ is of regular growth if there exist a compact neighborhood $U$ of $e$ and a constant $M$ so that

$$
|h(x)|<M \int_{U} h(x \cdot y) d y \quad \text { for all } x \in G .
$$

2.12 Proposition (SEe Gel'fand et Al. [GGP, p. 23]). Let $f \in C(G)$ and $h \in$ $C(G) \cap L^{1}(G)$ so that $|f|<h$ and $h$ has regular growth. Then the series $\Sigma_{\gamma} f\left(g^{-1} \gamma x\right)$ converges absolutely and uniformly on compacta of $G \times G$.

Any $x \in G$, can be written $x=k_{1} a k_{2}$ with $k_{1}, k_{2} \in K, a \in A$. Define $h(x)=$ $e^{-|\rho(\log a)|}$. Then $h(x)$ is well defined. Also $x=k \cdot \exp (X), k \in K, X \in \mathfrak{p}$ in a unique fashion. We define $\sigma(x)=B_{g}(X, X)^{1 / 2}$ where $B_{g}$ denotes as usual the Killing form of $g$.

2.13 Proposition. Let $r=$ split rank of $G$. Then for any $\varepsilon>0$

$$
f(x)=\frac{h(x)^{2}}{(1+\sigma(x))^{r+\varepsilon}}
$$

is of regular growth.

We refer to [GW] for a proof of the proposition.

2.14 Theorem. Let $f \in C^{3(n+1)}(G)$ be $K$-central, $K$-finite and such that $\Omega^{j} f \in$ $L^{1}(G)$ for $j=1,2, \ldots,(n+1)+[(n+1) / 2]$. Assume also that

$$
|f(x)|<\frac{h(x)^{2}}{(1+\sigma(x))^{r+e}}
$$

with $r=$ split rank of $G$ and $\varepsilon>0$. Then the Selberg trace formula (Theorem 2.1) is valid for $f$. 
Proof. The theorem is a direct consequence of 2.10, 2.12 and 2.13.

Let now $\phi_{0}(x)$ be the spherical function of weight 0 ,

$$
\phi_{0}(x)=\int_{K} e^{-\rho(H(x k))} d k
$$

where $H(x k)=\log a(x k)$ and $x k=K(x k) \cdot a(x k) \cdot n(x k)$ with $K(x k) \in K, a(x k)$ $\in A, n(x k) \in N$.

2.15 Definition. If $1<p<2$, let $\mathcal{C}^{p}(G)$ (the $p$-Schwartz space of $G$ ) be the set of all $C^{\infty}$ functions on $G$ such that for each natural $m$ and for each $D$, a product of a left invariant and a right invariant differential operator on $G$, there is a constant $M_{D, m}$ such that

$$
\sup _{x \in G}|D f(x)|<M_{D, m} \cdot \phi_{0}(x)^{2 / p} /(1+\sigma(x))^{m} .
$$

Then $\mathcal{C}^{p}(G)$ with the topology defined by the seminorms

$$
q_{D, m}(f)=\sup _{x \in G}\left[(1+\sigma(x))^{m} \cdot \phi_{0}(x)^{-2 / p} \cdot D f(x)\right]
$$

is a Fréchet space (cf. [HC1]). It is clear from the definitions that $f \in \mathcal{C}^{p}(G)$ implies that $\Omega^{k} f \in \mathcal{C}^{p}(G)$ for all $k$ and that $\mathcal{C}^{p}(G) \subset L^{p}(G)$ for any $p$.

2.16 Corollary. Let $f \in \mathcal{C}^{1}(G)$ be $K$-central and $K$-finite. Then $f$ goes into the Selberg trace formula.

3. The trace of the heat kernel. Let $G, K$ and $\Gamma$ be as in 1 and fix $\tau \in \hat{K}$. Consider the vector bundle $\Gamma \backslash G \times V_{\tau} \rightarrow \Gamma \backslash G / K$ over $M=\Gamma \backslash G / K$. We have a second order, elliptic, formally selfadjoint differential operator $D=-\nabla_{\Gamma}^{2}=-\Omega_{\Gamma}+\lambda_{\Gamma} \cdot I$ on $\Gamma \backslash G \times V_{\tau}$. The spectrum of $D$ is the sequence of eigenvalues $0=\lambda_{0}<\lambda_{1}<$ $\lambda_{2}, \ldots$ and $\lim _{i \rightarrow \infty} \lambda_{i}=\infty$. If $m_{i}=\operatorname{dim} C_{\lambda_{1}}^{\infty}\left(\Gamma \backslash G \times{ }_{\tau} V_{\tau}\right)$ we set

$$
\phi_{\tau, \Gamma}(s)=\phi_{\tau}(s)=\sum_{i} m_{i} \cdot e^{-\lambda \cdot s} \text { for } s>0 \text {. }
$$

We wish to study $\phi_{\tau}(s)$ near $s=0$. Wallach shows in [Wa3, Lemma 3.2] that $\phi_{\tau}(s)$ can be written

$$
\phi_{\tau}(s)=e^{-\lambda_{r} s} \sum_{\omega \in \hat{G}} n_{\Gamma}(\omega)\left[\tau: \omega_{\mid K}\right] \cdot e^{s \lambda_{\omega}}
$$

where $\lambda_{\tau}$ is as in Proposition 1.1 and $B_{g}\left(H_{1}, H_{1}\right)^{-1} \cdot \lambda_{\omega}$ is the eigenvalue of the Casimir element of $G$ on the class $\omega \in \hat{G}$.

Let us assume that there exists a function $f_{s}$ to which the Selberg trace formula applies and such that

$$
\theta_{\omega}\left(f_{s}\right)=\left[\tau: \omega_{\mid K}\right] \cdot e^{s\left(\lambda_{\omega}-\lambda_{r}\right)}
$$

It then follows that

$$
\operatorname{tr} \pi_{\Gamma}\left(f_{s}\right)=\sum_{\omega \in \hat{G}} n_{\Gamma}(\omega) \cdot \theta_{\omega}\left(f_{s}\right)=\phi_{\tau}(s)
$$

and on the other hand $\operatorname{tr} \pi_{\Gamma}\left(f_{s}\right)$ is given by this formula. Hence we can use the right-hand side of the formula applied to $f_{s}$ to study $\phi_{\tau}(s)$. 
Therefore there are two separate problems: to define such a function $f_{s}$ and then to study the right-hand side of the trace formula near $s=0$, for $s>0$. In the next section we will be concerned with the first of these questions.

4. The function $h_{\tau, s}$. Let $\left(\pi_{\sigma, \rho+i \nu}, \tilde{H}^{\sigma, \rho+i \nu}\right)$ be the principal series representations of $G$, parametrised as in [Wa1, Chapter 8]. Then $\pi_{\sigma, \rho+i \nu}$ is unitary if and only if $\nu \in a^{*}$. We write $\pi_{\sigma, \nu}=\pi_{\sigma, \rho+i \nu}$. Let $\hat{G}_{c}$ denote the set of all equivalence classes of irreducible constituents of unitary principal series representations. Also, let $\hat{G}_{d}$ be the discrete series of $G$, that is, those classes $\omega \in \hat{G}$ that contain a square integrable representation of $G$. We now state the version of the Plancherel theorem that we will need (see [HC2]).

4.1 THEOREM. Let $G$ be a connected Lie group with finite center and split rank one. Let $\lambda \in a^{*}$ be the long positive restricted root. Given $\sigma \in \hat{M}$, there exists a nonnegative, even function of polynomial growth $\mu_{o}(x \lambda)$ (for $\left.x \in \mathbf{R}\right)$ such that, for any $f \in \mathcal{C}^{2}(G), K$-finite on both sides,

$$
f(e)=\sum_{\omega \in \hat{G}_{d}} d(\omega) \cdot \theta_{\omega}(f)+\sum_{\sigma \in \hat{M}} \int_{-\infty}^{+\infty} \theta_{\pi_{o, x \lambda}}(f) \cdot \mu_{\sigma}(x \lambda) d x .
$$

Here $d(\omega)$ denotes the formal degree of $\omega \in \hat{G}_{d}\left(d(\omega)\right.$ and $\mu_{\sigma}(x \lambda)$ depend on the choice of the Haar measure on $G)$. Moreover all sums are finite.

Let $(\pi, H)$ be a unitary, irreducible representation of $G$. Then $(\pi, H)$ is $K$-finite, that is, $H$ is the unitary direct sum $H=\Sigma_{\tau \in \hat{K}} H_{\tau}$, where, as a representation of $K$, $\pi_{\mid H_{\tau}} \sim m_{\tau} \cdot \tau$ with $m_{\tau}<\infty$, for any $\tau \in \hat{K}$. We let $E_{\tau}: H \rightarrow H_{\tau}$ be the orthogonal projection onto $H_{\tau}$. We observe that if $d_{\tau} \cdot f * \chi_{\tau}=f$ then

$$
\theta_{\pi}(f)=\int_{G} \operatorname{tr}\left(E_{\tau^{*}} \cdot \pi(x) \cdot E_{\tau^{*}}\right) \cdot f(x) d x .
$$

If $(\pi, H)=\left(\pi_{\sigma, \nu}, H^{\sigma, \nu}\right)$, the space $H_{\tau}^{\sigma, \nu}$ is identified with $V_{\tau} \otimes \operatorname{Hom}_{M}\left(V_{\tau}, V_{\sigma}\right)$ via the map $A$ given by

$$
A(v \otimes T)(k a n)=e^{-(i v+\rho)(\log a)} \cdot T\left(\tau\left(k^{-1}\right) v\right) .
$$

The matrix entries of the principal series are given by Eisenstein integrals: if $\tau$, $\gamma \in \hat{K}, T \in \operatorname{Hom}_{M}\left(V_{\tau}, V_{\gamma}\right), \nu \in a_{C}^{*}$ and $x \in G$, let

$$
E_{\gamma, \tau}(T: i \nu: x)=\int_{K} e^{-(i \nu+\rho) H(x k)} \cdot \gamma(K(x k)) \cdot T \cdot \tau\left(k^{-1}\right) d k .
$$

Let $\left\{p_{\sigma}^{j} \mid j=1,2, \ldots, m_{\sigma}\right\}$ be a basis of $\operatorname{Hom}_{M}\left(V_{\tau}, V_{\sigma}\right)$.

A computation (see [Wa1, 8.12.4]) shows that

$$
\left\langle\pi_{\sigma, \nu}(x) A\left(w \otimes p_{\sigma}^{j}\right), A\left(w \otimes p_{\sigma}^{k}\right)\right\rangle=\left\langle E_{\tau, \tau}\left(p_{\sigma}^{j^{*}} \cdot p_{\sigma}^{k}:-i v: x\right) w, v\right\rangle .
$$

Let us fix $\tau \in \hat{K}$. Let $\hat{M}_{\tau}=\left\{\sigma \in \hat{M} \mid\left[\sigma: \tau_{\mid M}\right] \neq 0\right\}$. Then

$$
V_{\tau}=\sum_{\sigma \in \hat{M}_{\tau}} \sum_{j=1}^{m_{\sigma}} H_{\sigma}^{j} \quad \text { with }\left(\tau_{\mid M}, H_{\sigma}^{j}\right) \in \sigma .
$$

Let $q_{\sigma}^{j}=p_{\sigma}^{j *} \cdot p_{\sigma}^{j}$, for $j=1,2, \ldots, m_{\sigma}$. 
4.2 LemMA. For each $\sigma \in \hat{M}_{\tau}$ let $a_{\sigma}(\nu)$ be $a C^{\infty}$ function on $a_{\mathrm{C}}^{*}$ Set

$$
a_{\tau}(\nu)=\sum_{\sigma \in \hat{M}_{\tau}} \sum_{j=1}^{m_{\sigma}} a_{\sigma}(\nu) \cdot q_{\sigma}^{j} .
$$

Then

$$
\sum_{\sigma \in \hat{M}_{\tau}} \operatorname{tr}\left(E_{\tau} \pi_{\sigma, \nu}(x) E_{\tau}\right) a_{\sigma}(\nu)=\operatorname{tr} E_{\tau \tau}\left(a_{\tau}(\nu),-i \nu, x\right)
$$

for any $\nu \in a_{\mathbf{C}}^{*}$.

Proof. By the matrix expression for $E_{\tau} \cdot \pi_{\sigma, \nu}(x) \cdot E_{\tau}$ we have

$$
\begin{aligned}
& \sum_{\sigma \in \hat{M}_{\tau}} \operatorname{tr}\left(E_{\tau} \cdot \pi_{\sigma, \nu}(x) E_{\tau}\right) a_{\sigma}(\nu) \\
& \qquad \sum_{\sigma \in \hat{M}_{\tau}} \sum_{j=1}^{m_{\sigma}} \operatorname{tr} E_{\tau \tau}\left(q_{\sigma}^{j}:-i \nu: x\right) a_{\sigma}(\nu)=\operatorname{tr} E_{\tau \tau}\left(a_{\tau}(\nu):-i \nu: x\right) \text { Q.E.D. }
\end{aligned}
$$

Consider now the canonical double representation of $K$ on $\operatorname{End}\left(V_{\tau}\right)$. If $k_{1}, k_{2} \in$ $K$ and $T \in \operatorname{End}\left(V_{\tau}\right)$ let

$$
\mu\left(k_{1}, k_{2}\right)(T)=\tau\left(k_{1}\right) \cdot T \cdot \tau\left(k_{2}\right) .
$$

4.3 TheOREM [War, TheORem 9.1.5.1]. If $G$ is a rank one Lie group with finite center, there exist meromorphic $\operatorname{End}_{C}\left(\operatorname{End}_{M}\left(V_{\tau}\right)\right)$-valued functions on $a_{c}^{*}: c_{\tau}(\nu)$, $c_{-1, \tau}(\nu)$ and $\sigma(\nu: t)$ so that for any $T \in \operatorname{End}\left(V_{\tau}\right), \nu \in \mathrm{C}$ and $t>0$ we have

$$
\begin{aligned}
E_{\pi \tau}(T: & \left.i \nu \lambda: \exp t \frac{b}{2} \cdot H_{1}\right) \\
& =e^{-\|\rho\|(b / 2) t}\left(e^{i \nu b t} \cdot \sigma(\nu \lambda: t) \cdot c_{\tau}(\nu \lambda)+e^{-i \nu b t} \cdot \sigma(-\nu \lambda: t) \cdot c_{-1, \tau}(\nu \lambda)\right)(T) .
\end{aligned}
$$

Here, $b=$ number of distinct positive restricted roots (as in 2.1).

We now summarize some properties of $c_{\tau}(\nu), c_{-1, \tau}(\nu)$ and $\sigma(\nu: t)$ that we will need.

4.4 Proposition. There is a meromorphic function $c_{\tau}$ : $a_{C}^{*} \rightarrow \operatorname{End}_{M}\left(V_{\tau}\right)$ (that we still call $\left.c_{\tau}\right)$ such that for any $T \in \operatorname{End}_{M}\left(V_{\tau}\right)$,

$$
\begin{aligned}
c_{\tau}(\nu)(T) & =T \cdot c_{\tau}(\nu), \\
c_{-1, \tau}(\nu)(T) & =\tau\left(m_{0}\right)^{-1} \cdot c_{\tau}(\bar{\nu})^{*} \cdot T \cdot \tau\left(m_{0}\right) .
\end{aligned}
$$

Here $m_{0} \in M^{*}-M$ and $M^{*}=\{k \in K \mid \operatorname{Ad}(k)(a) \subset a\}$.

4.5 Proposition (Harish-Chandra [HC2, I, Lemma 13.4 and III, Theorem 27.3]). There exist constants $d_{\sigma} \in \mathbf{R}$ depending only on $\sigma$ so that, if

$$
b_{\tau}=\sum_{\sigma \in \hat{M}_{\tau}} \sum_{j=1}^{m_{0}} d_{\sigma} q_{\sigma}^{j}
$$


and if

$$
\mu_{\tau}(\nu)=\sum_{\sigma \in \hat{M}_{\tau}} \sum_{j=1}^{m_{0}} \mu_{\sigma}(\nu) q_{\sigma}^{j}
$$

then $c_{\tau}(\nu) \cdot c_{\tau}(\bar{\nu})^{*}=\mu_{\tau}(\nu)^{-1} \cdot b_{\tau}$.

4.6 Proposition. There exists a rational function $q_{1}(\nu)$ such that if $\operatorname{Im}\langle\nu, \alpha\rangle<0$,

$$
\left\|c_{\tau}(\nu)^{-1}\right\|<\left|q_{1}(\nu)\right|
$$

(\| || stands for the operator norm in $\operatorname{End}\left(V_{\tau}\right)$ ).

We turn our attention to the function $\sigma(\nu: t)$ of 4.3. As shown in Warner [War, Chapter 9], this function is given by a series

$$
\sigma(\nu: t)=\sum_{k=0}^{\infty} \Gamma_{k}(i \nu-\rho) e^{-k t}
$$

where the $\Gamma_{k}(i \nu-\rho)$ are rational functions on $a_{C}^{*}$ with values in $\operatorname{End}_{C}\left(\operatorname{End}_{M} V_{\tau}\right)$. These functions are defined by means of complicated recurrence relations which we will not need to describe. We will make use of the following important result.

4.7 Proposition. There is a rational function $q_{2}(\nu)$ and a constant $\dot{c}$ such that if $\nu \in a_{\mathrm{C}}^{*}$ and $\operatorname{Im}\langle\nu, \lambda\rangle>0$ then $\left\|\Gamma_{k}(i \nu-\rho)\right\|\left\langle\left|q_{2}(\nu)\right| \cdot c^{k}, k=0,1,2, \ldots\right.$

Proof. This estimate is contained in the proof of Theorem 2.4 in Chapter II of Helgason [He]. See also Johnson [J, Lemma 2.1].

Now let $X_{1}, X_{2}, \ldots, X_{m}$ be a basis of $m$ such that $\left\langle X_{i}, X_{j}\right\rangle=-\delta_{i j}$ and let

$$
\omega_{M}=-\sum_{i} X_{i}^{2}
$$

Then, since $\omega_{M}$ is a scalar multiple of the Casimir element of $M$, given $\sigma \in \hat{M}$ there is $\lambda_{\sigma}$ such that $\sigma\left(\omega_{M}\right)=\lambda_{\sigma} \cdot I$.

4.8 Proposition. The poles of $\Gamma_{k}(i \nu \lambda-\rho)$ lie in the set

$$
P_{k, \tau}=\left\{\nu \in \mathbf{C} \mid \nu=\frac{-i}{2 b}\left(h+\frac{\lambda_{\sigma_{i}}-\lambda_{o_{j}}}{h}\right) \text { where } h=1,2, \ldots, k \text { and } \sigma_{i}, \sigma_{j} \subset \tau_{\mid M}\right\} \text {. }
$$

Proof. In the notation of Warner [War, 9.1.4] we let $\tau\left(\omega_{M}\right) \in \operatorname{End}\left(\operatorname{End}_{M}\left(V_{\tau}\right)\right)$ be given by

$$
\tau\left(\omega_{M}\right)(T)=T \cdot \tau\left(\omega_{M}\right)
$$

If $V_{\tau}=\Sigma_{\sigma \in \hat{M}_{\tau}} \sum_{j=1}^{m_{0}} H_{\sigma}^{j}$ and $q_{\sigma}^{j}: V_{\tau} \rightarrow H_{\sigma}^{j}$ is orthogonal projection onto $H_{\sigma}^{j}$ we have that $\tau\left(\omega_{M}\right)\left(q_{\sigma}^{j}\right)=\lambda_{\sigma} \cdot q_{\sigma}^{j}$. Hence, the eigenvalues of

$$
\gamma=\operatorname{ad}\left(\tau\left(\omega_{M}\right)\right): \operatorname{End}\left(\operatorname{End}\left(V_{\tau}\right)\right) \rightarrow \operatorname{End}\left(\operatorname{End}\left(V_{\tau}\right)\right)
$$

are all the differences $\lambda_{o_{i}}-\lambda_{\sigma_{j}}$ with $\sigma_{i}, \sigma_{j} \in \hat{M}_{\tau}$. By the very definition of $\Gamma_{k}$, its poles lie in the set $\left\{\nu \in a_{c}^{*} \mid 2 h \cdot B_{g}\left(H_{\alpha}, i H_{\nu}\right)=h^{2} \cdot B_{g}\left(H_{\alpha}, H_{\alpha}\right)+\gamma_{j} B_{g}\left(H_{\alpha}, H_{\alpha}\right)\right.$ where $h=1,2, \ldots, k$ and $\gamma_{j}$ runs over all eigenvalues of $\gamma$ \}. Here $\alpha$ denotes the short positive restricted root, that is $\alpha=\lambda / b$. Hence, the poles of $\Gamma_{k}(i \nu \lambda-\rho)$ lie in 
the set

$$
\begin{aligned}
P_{k, \tau}=\left\{\nu \mid \nu \in \mathrm{C} \text { and } \nu=(-i / 2 b) \cdot\left(h+\frac{\lambda_{\sigma_{i}}-\lambda_{\sigma_{j}}}{h}\right)\right. \\
\left.\quad \text { with } h=1,2, \ldots, k \text { and } \sigma_{i}, \sigma_{j} \in \hat{M}_{\tau}\right\} . \text { Q.E.D. }
\end{aligned}
$$

We now prove an estimate that we will need later on.

4.9 Proposition. Let $f(z)$ be a meromorphic function on $\bar{H}=[z \mid \operatorname{Im} z>0]$ such that $|f(z)|<|q(z)|$ for $z \in \bar{H}, q(z)$ being a rational function of degree $N$. Suppose also that the poles $z_{1}, z_{2}, \ldots, z_{k}$ of $f$ in $\bar{H}$ are off the real axis, and that $N_{j}$ is the order of the pole $z_{j}$. If $a>0$ is a constant we put

$$
I(t)=\int_{-\infty}^{+\infty} e^{-a x^{2}} e^{i t x} f(x) d x
$$

Then there exist polynomials $p_{j}(t)$ of degree $N_{j}-1$ for $j=1,2, \ldots, k$ so that

$$
\exp \left(-t^{2} / 4 a\right) \cdot\left(I(t)-\sum_{j=1}^{k} p_{j}(t) \cdot e^{i z j t}\right)=O\left(t^{N}\right) \text { as } t \rightarrow+\infty \text {. }
$$

Proof. Since we are interested in large values of $t$ we may assume that $t / 2 a>\operatorname{Im} z_{j}$, for $j=1,2, \ldots, k$. Let $x=y \cdot t$. Then

$$
I(t)=t \cdot \int_{-\infty}^{+\infty} e^{-t^{2}\left(a y^{2}-y i\right)} f(y t) d y .
$$

We use the method of steepest descent to study $I(t)$. If $h(y)=a y^{2}-i y$, then $h^{\prime}(y)=2 a y-i$. We move the contour of integration up to the line $\operatorname{Im} y=1 / 2 a$. Since the poles of $f(y t)$ are at $z_{j} / t$ and $t / 2 a>\operatorname{Im} z_{j}$ we have

$$
\begin{aligned}
I(t)= & t \int_{-\infty}^{+\infty} e^{-t^{2}\left(a u^{2}+1 / 4 a\right)} \cdot f\left(u t+\frac{i t}{2 a}\right) d u \\
& +2 \pi i t \sum_{j=1}^{k} \underset{y=z_{j} / t}{\operatorname{Res}} g(y),
\end{aligned}
$$

where $g(y)=e^{-t^{2}\left(a y^{2}-i y\right)} f(y t)$.

Letting $u t=x$,

$$
I(t)=e^{-t^{2} / 4 a} \int_{-\infty}^{+\infty} e^{-a x^{2} f}\left(x+\frac{i t}{2 a}\right) d x+2 \pi i t \sum_{j=1}^{k} \underset{\substack{\operatorname{Res} \\ y=z_{j} / t}}{\operatorname{Re}} g(y) .
$$

Since for $z \in \bar{H},|z|$ large, $|f(z)|<c|z|^{N}$ it is clear that

$$
\int_{-\infty}^{+\infty} e^{-a x^{2}} f\left(x+\frac{i t}{2 a}\right) d x=O\left(t^{N}\right) \text { as } t \rightarrow+\infty \text {. }
$$

On the other hand, if $h(z)=e^{-t^{2}\left(a z^{2}-i z\right)}$ then a computation shows that

$$
\frac{1}{l !} h^{(l)}\left(\frac{z_{j}}{t}\right)=\tilde{P}_{l, j}(t) \cdot t^{l} e^{i z z_{j}}
$$

where $\tilde{P}_{l, j}(t)$ is a polynomial of degree $l$. 
Thus, if $f(z)=\Sigma_{-N_{j}}^{\infty} b_{i j}\left(z-z_{j}\right)^{i}$,

$$
\begin{aligned}
2 \pi i t \underset{z=z_{j} / t}{\operatorname{Res}} g(z) & =2 \pi i t\left(\sum_{l=0}^{N_{j}-1} \frac{1}{l !} h^{(l)}\left(\frac{z_{j}}{t}\right) \cdot b_{-l-1, j} t^{-l-1}\right) \\
& =\left(\sum_{l=0}^{N_{j}-1} 2 \pi i \cdot b_{-l-1, j} \tilde{P}_{l, j}(t)\right) e^{i t z_{j}} .
\end{aligned}
$$

This completes the proof of the proposition.

We next use a wave packet to define a function $h_{\tau, s}$ closely related to our specific problem (see §3). Our main task will be to study the growth of this function.

4.10 Definition. If $\tau \in \hat{K}$ and $s>0$ we set

$$
h_{\tau, s}(s)=d_{\tau}^{-1} \cdot e^{-\lambda_{r} \cdot s} \cdot \sum_{\sigma \in \hat{M}_{\tau}} \int_{-\infty}^{+\infty} \operatorname{tr}\left(E_{\tau} \cdot \pi_{\sigma, \nu \lambda}(x) \cdot E_{\tau}\right) e^{s \lambda_{,}, \nu \lambda} \cdot \mu_{\sigma}(\nu \lambda) d \nu
$$

where $\mu_{0}$ is as in 4.1.

Here $\lambda_{o, \nu \lambda}$ denotes the eigenvalue of the Casimir $\Omega_{\langle,\rangle}=B_{g}\left(H_{1}, H_{1}\right) \cdot \Omega$ on the class $\pi_{\sigma, \nu \lambda}$. Let $\alpha$ denote the short positive restricted root. Then $b \alpha=\lambda$ where, as usual, $b$ is the number of distinct positive restricted roots. By the computation in [Wa1, p. 280] we have

$$
B_{\mathrm{g}}\left(H_{1}, H_{1}\right)^{-1} \cdot \lambda_{\sigma, i z \rho}=z^{2} \cdot \frac{\rho\left(H_{\alpha}\right)^{2}}{\alpha\left(H_{\alpha}\right)}-\frac{\rho\left(H_{\alpha}\right)}{\alpha\left(H_{\alpha}\right)}-\lambda_{\sigma} .
$$

Since $\lambda=b \alpha$ and $z \rho=-i \nu \lambda$ we have $z=-2 i \nu / \rho\left(H_{1}\right), H_{1}=2 H_{\alpha} / b \cdot \alpha\left(H_{\alpha}\right)$. Thus

$$
\lambda_{\sigma, i z \rho}=-B_{\mathrm{g}}\left(H_{1}, H_{1}\right) \cdot \alpha\left(H_{\alpha}\right) \cdot\left[b^{2} \nu^{2}+\frac{\rho\left(H_{\alpha}\right)^{2}}{\alpha\left(H_{\alpha}\right)}+\frac{\lambda_{\sigma}}{\alpha\left(H_{\alpha}\right)}\right] \text {. }
$$

But $\|\rho\|^{2}=\left\langle\tilde{H}_{\rho}, \tilde{H}_{\rho}\right\rangle$, where $\tilde{H}_{\rho}$ satisfies $\rho(H)=B_{g}\left(\tilde{H}_{\rho}, H\right) / B_{g}\left(H_{1}, H_{1}\right)$, that is, $\tilde{H}_{\rho}=B_{\mathrm{g}}\left(H_{1}, H_{1}\right) \cdot H_{\rho}$. Thus $\|\rho\|^{2}=B_{g}\left(H_{1}, H_{1}\right) \cdot B_{g}\left(H_{\rho}, H_{\rho}\right)$. Using that $B_{g}\left(H_{\alpha}, H_{\alpha}\right) \cdot B\left(H_{1}, H_{1}\right)=4 / b^{2}$ we finally get that

$$
\|\rho\|^{2}=\frac{4}{b^{2}} \cdot \frac{\rho\left(H_{\alpha}\right)^{2}}{\alpha\left(H_{\alpha}\right)} .
$$

Hence the formula for the eigenvalue is

$$
\lambda_{\sigma, \nu \lambda}=-\left(4 \nu^{2}+\|\rho\|^{2}+B_{g}\left(H_{1}, H_{1}\right) \cdot \lambda_{\sigma}\right) .
$$

Thus, letting $a=B_{\mathrm{g}}\left(H_{1}, H_{1}\right)$,

$$
\begin{aligned}
h_{\tau, s}(x)= & d_{\tau}^{-1} \cdot e^{-\left(\lambda_{r}+\|\rho\|^{2}\right) s} \\
& \cdot \int_{-\infty}^{+\infty} \sum_{\sigma \in \hat{M}_{\tau}} \operatorname{tr}\left(E_{\tau} \cdot \pi_{\sigma, \nu \lambda}(x) E_{\tau}\right) \cdot e^{-s \lambda_{\sigma} a} \cdot e^{-s \cdot 4 \nu^{2}} \cdot \mu_{\sigma}(\nu \lambda) d \nu .
\end{aligned}
$$

Let $e_{\tau, s}=\Sigma_{\sigma \in \hat{M}_{\tau}} \sum_{j=1}^{m_{0}} e^{-s \lambda_{-} \cdot a} \cdot q_{\sigma}^{i} \in \operatorname{End}_{M}\left(V_{\tau}\right)$. Then, by 4.2

$$
h_{\tau, s}(x)=d_{\tau}^{-1} \cdot e^{-\left(\lambda_{r}+\|\rho\|^{2}\right) s} \cdot \int_{-\infty}^{+\infty} \operatorname{tr} E_{\tau \tau}\left(\mu_{\tau}(\nu \lambda) \cdot e_{\tau, s}:-i \nu \lambda: x\right) e^{-4 v \nu^{2}} d \nu
$$


Let us set $a_{t}^{\prime}=\exp \left(t \cdot b H_{1} / 2\right)$. Using 4.3, 4.4 and $4.5 h_{\tau, 5}\left(a_{t}^{\prime}\right)$ can be put into the following form

$$
\begin{aligned}
h_{\tau, s}\left(a_{t}^{\prime}\right)= & d_{\tau}^{-1} \cdot e^{-\left(\lambda+\|\rho\|^{2}\right) s} \cdot e^{-\|\rho\| b \cdot t / 2} \\
& \cdot \int_{-\infty}^{+\infty} e^{-4 s \nu^{2}} \operatorname{tr}\left[e^{i \nu b t} \cdot \sigma(\nu \lambda: t) \cdot b_{\tau} \cdot e_{\tau, s} \cdot c_{\tau}(\bar{\nu} \lambda)^{*-1}\right. \\
& \left.\quad+e^{-i \nu b t} \cdot \sigma(-\nu \lambda: t) \tau\left(m_{0}\right)^{-1} \cdot c_{\tau}(\nu \lambda)^{-1} \cdot b_{\tau} \cdot e_{\tau, s} \cdot \tau\left(m_{0}\right)\right] d \nu .
\end{aligned}
$$

We now need the fact that the poles of $\sigma(\nu \lambda: t)$ and $c_{\tau}(\bar{\nu} \lambda)^{*-1}$ form a discrete subset of the imaginary axis. For $c_{\tau}(\bar{\nu} \lambda)^{*-1}$ this is a consequence of $4.5,4.6$ and of the expression of the matrix entries of $c_{\tau}(\nu \lambda)$ as linear combinations of products of beta functions (see [Wa4]). Moreover, only finitely many of those poles: $i \nu_{1}, \ldots, i \nu_{l}$ lie in $\bar{H}$, the closed upper half plane. To avoid the possible pole at $\nu=0$, we change the contour of integration near $\nu=0$. That is, the new contour $\Gamma_{e}$ consists of the half-lines $L_{\varepsilon}^{+}=\{\nu \mid \nu \geqslant \varepsilon\}, L_{e}^{-}=\{\nu \mid \nu<-\varepsilon\}$ and the semicircle $C_{e}=\{\nu=$ $\left.\varepsilon \cdot e^{i t} \mid 0<t \leqslant \pi\right\}$. We choose $\varepsilon$ so that the new contour will be within the strip of holomorphy of the integrand and also so that the only pole of $\sigma(\nu \lambda: t)$ or $c_{\tau}(\bar{\nu} \lambda)^{*-1}$ inside the circle of center at $\nu=0$ and radius $\varepsilon$ is, possibly, $\nu=0$.

Then we have that the only poles of the first half of the integrand that lie above $\Gamma_{e}$ are $i \nu_{1}, i \nu_{2}, \ldots, i \nu_{l}$ (where $\nu_{j}=0$, all $j$ ) and since the poles of $\sigma(-\nu \lambda: t)$ and $c_{\tau}(\nu \lambda)^{-1}$ are the negative of the poles of $\sigma(\nu \lambda: t)$ and $c_{\tau}(\bar{\nu} \lambda)^{*-1}$ respectively, and because of the choice of $\varepsilon$, the poles of the second half of the integrand that lie below $\Gamma_{e}$ are $-i \nu_{1},-i \nu_{2}, \ldots, i \nu_{l}$ and possibly $\nu=0$.

To estimate the growth of $h_{\tau, s}\left(a_{t}^{\prime}\right)$ for $t$ large we use Proposition 4.9. We note that we need a slightly different formulation of it, since the contour of integration is $\Gamma_{e}$ instead of the real axis and moreover because we will move the contour below $\Gamma_{e}$ to study the second half of the integrand. However, making the obvious changes in the proof we see that the proposition is still valid. We now break the integral into two pieces.

$$
\begin{aligned}
& \int_{\Gamma_{e}} e^{-4 s \nu^{2}} e^{i \nu b t} \operatorname{tr} \sigma(\nu \lambda: t) b_{\tau} \cdot e_{\tau, s} c_{\tau}(\bar{\nu} \lambda)^{*-1} d \nu \\
&=\int_{\Gamma_{e}} e^{-4 s \nu^{2}} e^{i \nu b t} \operatorname{tr}\left(\sum_{k} \Gamma_{k}(i \nu \lambda-\rho) e^{-k t} b_{\tau} \cdot e_{\tau, s} c_{\tau}(\bar{\nu} \lambda)^{*-1}\right) d \nu
\end{aligned}
$$

By 4.6 and 4.7 there is a rational function $\tilde{q}(\nu)$ and a constant $c$ so that

$$
\left|\operatorname{tr} \Gamma_{k}(i \nu \lambda-\rho) b_{\tau} \cdot e_{\tau, s} c_{\tau}(\bar{\nu} \lambda)^{*-1}\right|<|\tilde{q}(\nu)| c^{k} \quad \text { if } \nu \in \bar{H} \text {. }
$$

Therefore the integrand is dominated by $\left|e^{i v b t} e^{-4 s \nu^{2}} \cdot \tilde{q}(\nu) /\left(1-c e^{-t}\right)\right|$, if $t>1$ and dominated convergence applies. Hence the above is

$$
=\sum_{k=0}^{\infty} \int_{\Gamma_{e}} e^{i \nu b t} e^{-4 s \nu^{2}} \operatorname{tr}\left(\Gamma_{k}(i \nu \lambda-\rho) b_{\tau} \cdot e_{\tau, s} c_{\tau}(\bar{\nu} \lambda)^{*-1}\right) d \nu e^{-k t}
$$

Now let be (see Proposition 4.8)

$$
P_{k, \tau}^{+}=\left\{\nu \in H \mid \nu=\frac{-i}{2 b}\left(h+\frac{\lambda_{\sigma_{i}}-\lambda_{\sigma_{j}}}{h}\right), h=1,2, \ldots, k \text { and } \sigma_{i}, \sigma_{j} \in \hat{M}_{\tau}\right\}
$$


Then $P_{0, \tau}^{+}=\varnothing$ and

$$
P_{0, \tau}^{+} \subset P_{1, \tau}^{+} \subset \ldots \subset P_{k, \tau}^{+} \subset \ldots
$$

and this chain is stationary if $k$ is sufficiently large. We also put

$$
\begin{aligned}
& P_{\tau}^{+}=\cup{ }_{k=1}^{\infty} P_{k, \tau}^{+}, \\
& Z_{\tau}^{+}=\left\{\nu \in H \mid c_{\tau}(\bar{\nu} \cdot \lambda)=0\right\}, \\
& \tilde{P}_{k, \tau}=P_{k, \tau}^{+} \cup Z_{\tau}^{+}, \\
& \tilde{P}_{\tau}=P_{\tau}^{+} \cup Z_{\tau}^{+} .
\end{aligned}
$$

Let $\tilde{P}_{\tau, k}=\left\{i \nu_{j} \mid j=1,2, \ldots, l_{k}\right\}$ and $\tilde{P}_{\tau}=\left\{i \nu_{j} \mid j=1,2, \ldots, l\right\}$. It will be convenient to assume that

$$
\begin{aligned}
& Z_{\tau}^{+}=\left\{i \nu_{j} \mid j=1,2, \ldots, l_{0} \text { and } 0<\nu_{1}<\nu_{2}<\ldots<\nu_{l_{0}}\right\}, \\
& P_{\tau, k}^{+}=\left\{i \nu_{j} \mid j=l_{0}+1, \ldots, l_{k} \text { and } \nu_{l_{0}+1}>\nu_{l_{0}+2}>\ldots>\nu_{l_{k}}\right\}, \\
& P_{\tau}^{+}=\left\{i \nu_{j} \mid j=l_{0}+1, \ldots, \text { land } \nu_{l_{0}+1}>\nu_{l_{0}+2}>\ldots>\nu_{l}\right\} .
\end{aligned}
$$

Also let $N_{j, k}$ be the order of $i \nu_{j}$ as a pole of $\operatorname{tr} \Gamma_{k}(i \nu \lambda-\rho) b_{\tau} e_{\tau, s} c_{\tau}(\bar{\nu} \lambda)^{*-1}$. If we apply Proposition 4.9 to our integral we find that there exist polynomials $p_{j, k}(t)$ of degree $N_{j, k}-1$ and functions $g_{k}(t)$ so that

$$
\begin{aligned}
\sum_{k=0}^{\infty} \int_{\Gamma_{e}} e^{i \nu b t} & \cdot e^{-4 s \nu^{2}} \cdot \operatorname{tr}\left(\Gamma_{k}(i \nu \lambda-\rho) b_{\tau} \cdot e_{\tau, s} c_{\tau}(\bar{\nu} \lambda)^{*-1}\right) e^{-k t} \\
& =\sum_{k=0}^{\infty} \sum_{j=1}^{l_{k}} p_{j, k}(t) \cdot e^{-\left(\nu_{j}+k\right) t}+\exp \left(-b^{2} t^{2} / 16 s\right) \cdot \sum_{k=0}^{\infty} g_{k}(t) e^{-k t} .
\end{aligned}
$$

Moreover $\left|g_{k}(t)\right|<c^{k} \cdot O\left(t^{N}\right)$ as $t \rightarrow+\infty$, with $N=\operatorname{deg}(\tilde{q}(\nu))$.Thus,

$$
\left|\sum g_{k}(t) e^{-k t}\right|<\frac{1}{1-c e^{-t}} \cdot O\left(t^{N}\right)=O\left(t^{N}\right) \text { as } t \rightarrow+\infty .
$$

For the other half of the integral we must use the region below $\Gamma_{e}$. Hence the possible poles of the integrand are $\nu=-i \nu_{j}$ with $i \nu_{j} \in \tilde{P}_{\tau}$ or $\nu=0$. By Proposition 4.9 there exist polynomials $\tilde{p}_{j, k}(t)$ of degree $N_{j, k}-1$ and functions $\tilde{g}_{k}(t)$ so that

$$
\begin{aligned}
\int_{\Gamma_{e}} \sum_{k} e^{-i b \nu t} e^{-4 s \nu^{2}} & \operatorname{tr}\left(\Gamma_{k}(-i \nu \lambda-\rho) \tau\left(m_{0}^{-1}\right) c_{\tau}(\nu \lambda)^{-1} b_{\tau} e_{\tau, s} \tau\left(m_{0}\right)\right) d \nu \\
= & \sum_{k=0}^{\infty} \sum_{j=0}^{l_{k}} \tilde{p}_{j, k}(t) e^{-\left(\nu_{j}+k\right) t}+\sum_{k=0}^{\infty} \tilde{g}_{k}(t) e^{-k t} \cdot \exp \left(-b^{2} t^{2} / 16 s\right) .
\end{aligned}
$$

In this case summation starts at $j=0$ corresponding to the possible pole at $\nu_{0}=0$. We observe that since 0 is not a pole of $c_{\tau}(\nu \lambda)^{-1}$ and by definit; $\Gamma_{0}(i \nu \lambda-\rho) \equiv 1$, we have $\tilde{p}_{0,0}(t)=0$.

The next result summarises the properties of $h_{\tau, s^{*}}$.

4.11 Theorem. Fix $\tau \in \hat{K}$. Let

$$
h_{\tau, s}(x)=d_{\tau}^{-1} e^{-s \lambda} \sum_{\sigma \in \hat{M}_{\tau}} \int_{a^{*}} \operatorname{tr}\left(E_{\tau} \pi_{\sigma, \nu}(x) E_{\tau}\right) e^{s \lambda_{\sigma, \nu} \mu_{\sigma}(\nu) d \nu}
$$


where $a^{*}$ is parametrised by $\lambda$ and $\mu_{\sigma}$ is as in 4.1. Then

(i) $d_{\tau} \chi_{\tau} * h_{\tau, s}=d_{\tau} h_{\tau, s} * \chi_{\tau}=h_{\tau, s}$,

(ii) $h_{\tau, s} \in \mathcal{C}^{2}(G)$, the 2-Schwartz space of $G$,

(iii) $\theta_{\pi_{\sigma, p}}\left(h_{\tau, s}\right)=\left[\sigma: \tau_{\mid M}\right] e^{s\left(\lambda_{0, \nu}-\lambda_{1}\right)}$,

(iv) $h_{\tau, s}=h_{\tau, s}^{0}+h_{\tau, s}^{1}$

where $h_{\tau, s}^{0}$ is a linear combination of matrix entries of the discrete series, $h_{\tau, s}^{1} \in \mathcal{C}^{p}(G)$, the p-Schwartz space of $G$, for all $p>0$, and both $h_{\tau, s}^{0}$ and $h_{\tau, s}^{1}$ satisfy (i).

Proof. Parts (i) and (iii) are clear from the definition and the Plancherel theorem. By the estimates we just proved, we see that there exist polynomials $q_{j, k}(t)$ such that

$$
\begin{aligned}
h_{\tau, s}\left(a_{t}\right) & =e^{-\left(\lambda+\|\rho\|^{2}\right) s} \\
\cdot & e^{-\|\rho\| t}\left[\sum_{k=0}^{\infty} \sum_{j=0}^{l_{k}} q_{j, k}(t) \cdot e^{-\left(v_{j}+k\right) 2 t / b}+\exp \left(-t^{2} / 4 s\right) \cdot O\left(t^{N}\right)\right], \quad \text { as } t \rightarrow+\infty,
\end{aligned}
$$

where $a_{t}=\exp \left(t H_{1}\right)$.

Since $q_{0,0}=0, e^{\|\rho\|} \cdot h_{\tau, s}\left(a_{t}\right)$ decays exponentially as $t \rightarrow+\infty$. Since $d g=$ $c\left(\sinh ^{4} 2 t / b\right)^{m_{\alpha}} \cdot\left(\sinh ^{4} t / b\right)^{m_{2 a}} d k_{1} d t d k_{2}$, this says that $(1+\sigma(x))^{m} \cdot h_{\tau, s}(x) \in L^{2}(G)$ for all $m \in \mathbf{Z} \geqslant 0$. On the other hand

$$
\begin{aligned}
\Omega^{k} h_{\tau, s}(x)= & d_{\tau}^{-1} \cdot e^{-\left(\lambda_{r}+\|\rho\|^{2}\right) s} \\
& \cdot \operatorname{tr} \int_{-\infty}^{+\infty} \Omega^{k} E_{\tau \tau}\left(\mu_{\tau}(\nu \lambda) e_{\tau, s}:-i \nu \lambda: x\right) e^{-4 s \nu^{2}} d \nu \\
= & d_{\tau}^{-1} \cdot e^{-\left(\lambda_{r}+\|\rho\|^{2}\right) s} \\
& \cdot \operatorname{tr} \int_{-\infty}^{+\infty}(-1)^{k} E_{\tau \tau}\left(\sum_{\sigma, j} \mu_{\sigma}(\nu \lambda) e^{-s \lambda_{\sigma} a}\left(4 \nu^{2}+\|\rho\| \lambda_{\sigma}\right)^{k} q_{\sigma}^{j}:-i \nu \lambda: x\right) e^{-4 s \nu^{2}} d \nu
\end{aligned}
$$

Therefore, arguing as in the case of $h_{\tau, s}$ we get a similar estimate to show that $(1+\sigma(x))^{m} \Omega^{k} h_{\tau, s}(x) \in L^{2}(G)$ for all $m, k \in \mathbf{Z}>0$. Hence $h_{\tau, s} \in \mathcal{C}^{2}(G)$. Finally let us consider the above expression for $h_{\tau, s}\left(a_{t}\right)$. Campoli has shown (essentially, see [C]) that the first summand is the restriction to $A$ of $h_{\tau, s}^{0}$, a linear combination of matrix entries of the discrete series. From the expression of $h_{\tau, s}-h_{\tau, s}^{0}$ on $A$ (the second summand) we see that $h_{\tau, s}^{1}=h_{\tau, s}-h_{\tau, s}^{0} \in \mathcal{C}^{p}(G)$, for all $p>0$. Q.E.D.

The main difficulty at this point is the fact that we need $(1+\sigma(x))^{m} \Omega^{k} h_{\tau, s}(x) \in$ $L^{1}(G)$ for all $m$ and $k \in Z>0$ (instead of $(1+\sigma(x))^{m} \Omega^{k} h_{\tau, s}(x) \in L^{2}(G)$ ) in order for $h_{\tau, s}$ to be plugged into the right-hand side of the Selberg trace formula (see 2.1). This is false in general and we see from the proof of Theorem 4.10 that the obstruction comes from those poles $i z_{j}, z_{j}>0$, of $\operatorname{tr}\left(\sigma(\nu \lambda: t) b_{\tau} e_{\tau, s} c_{\tau}(\bar{\nu} \lambda)^{*-1}\right)$ that occur as a pole of $\operatorname{tr}\left(\Gamma_{k}(i \nu \lambda-\rho) b_{\tau} e_{\tau, s} c_{\tau}(\bar{\nu} \lambda)^{*-1}\right)$ for some $k$ so that $k+z_{j}<\|\rho\|$. $b / 2$. Let $i z_{1}, i z_{2}, \ldots, i z_{r}$ be these poles and let $N_{j}$ be the order of $i z_{j}$ as a pole of $\operatorname{tr}\left(\sigma(\nu \lambda: t) b_{\tau} e_{\tau, s} c_{\tau}(\bar{\nu} \lambda)^{*-1}\right)$. Set

$$
p(\lambda)=\prod\left\{\left(\lambda-\lambda_{\xi}, i z_{j}\right)^{N_{j}} \mid \xi \in \hat{M}_{\tau} \text { and } j=1, \ldots, r\right\} .
$$


4.12 TheOREM. If $\tau \in \hat{K}$ and $s>0$, let

$$
\tilde{h}_{\tau, s}(x)=d_{\tau}^{-1} \cdot e^{-\lambda_{\tau} s} \sum_{\sigma \in \hat{M}_{\tau}} \int_{a^{*}} \operatorname{tr}\left(E_{\tau} \pi_{\sigma, \nu}(x) E_{\tau}\right) p\left(\lambda_{\sigma, \nu}\right) e^{s \lambda_{, . \nu} \mu_{\sigma}(\nu) d \nu .}
$$

Then,

(i) $d_{\tau} \chi_{\tau} * \tilde{h}_{\tau, s}=d_{\tau} \tilde{h}_{\tau, s} * \chi_{\tau}=\tilde{h}_{\tau, s}$.

(ii) $\tilde{h}_{\tau, s} \in \mathrm{e}^{\mathrm{l}}(G)$.

(iii) $\theta_{\pi_{\sigma, v}}\left(\tilde{h}_{\tau, s}\right)=\left[\sigma: \tau_{\mid M}\right] e^{s\left(\lambda_{0, \nu}-\lambda_{1}\right)} p\left(\lambda_{o, v}\right)$.

Proof. Parts (i) and (iii) are clear. The argument in 4.11 when applied to $\tilde{h}_{\tau, s}$ gives a similar estimate for $\tilde{h}_{\tau, s}\left(a_{t}\right), t$ large. But the poles $i \nu_{j} \in H$ arising in the proof, satisfy $\nu_{j}+k>\|\rho\| b / 2$, hence $\tilde{h}_{\tau, s} \in L^{1}(G)$. Similarly we see that $\tilde{h}_{\tau, s} \in$ $\mathrm{e}^{\mathrm{l}}(G)$.

5. Asymptotic estimates for $\phi_{\tau}(s)$. By Theorem 4.12 the function $\tilde{h}_{\tau, s} \in \mathcal{C}^{\prime}(G)$. Hence, by Corollary $2.16, \operatorname{tr} \pi_{\Gamma}\left(\tilde{h}_{\tau, s}\right)$ can be evaluated by the right-hand side of the Selberg trace formula. Theorem 2.1 implies

$$
\begin{aligned}
\operatorname{tr} \pi_{\Gamma}\left(\tilde{h}_{\tau, s}\right)= & \operatorname{vol}(\Gamma \backslash G) \cdot \tilde{h}_{\tau, s}(e)+\frac{1}{2 \pi} \sum_{[\gamma] \in[\Gamma]-[e]} \operatorname{vol}\left(\Gamma_{\gamma} \backslash G_{\gamma}\right) D(\gamma)^{-1} \\
& \cdot u(\gamma)^{-1} \sum_{\sigma \in \hat{M}_{\tau}} b \cdot \overline{\chi_{0}\left(m_{\gamma}\right)} \int_{-\infty}^{+\infty} \theta_{\pi_{0,0 \lambda}}\left(\tilde{h}_{\tau, s}\right) \cdot e^{-2 i i_{\gamma}, \nu} d \nu
\end{aligned}
$$

We now apply results of De George to show that the right-hand side is asymptotic to $\operatorname{vol}(\Gamma \backslash G) \cdot \tilde{h}_{\tau, s}(e)$ as $s \downarrow 0$.

5.1 TheOREM. Let $\varepsilon(s)=\operatorname{tr} \pi_{\Gamma}\left(\tilde{h}_{\tau, s}\right)-\operatorname{vol}(\Gamma \backslash G) \tilde{h}_{\tau, s}(e)$. Then there exists $d>0$ such that

$$
\lim _{\substack{s \rightarrow 0 \\ s>0}} \varepsilon(s) \cdot e^{(d / s)}=0 .
$$

In particular this says that $\varepsilon(s)$ can be extended to a $C^{\infty}$-function on $\mathbf{R}$ by setting $\varepsilon(s)=0$ if $s<0$.

Proof.

$$
\begin{aligned}
\varepsilon(s)= & \frac{1}{2 \pi} \sum \operatorname{vol}\left(\Gamma_{\gamma} \backslash G_{\gamma}\right) D(\gamma)^{-1} u(\gamma)^{-1} \sum \overline{\chi_{\sigma}\left(m_{\gamma}\right)} \\
& \cdot b \cdot\left[\sigma: \tau_{\mid M}\right] e^{-\left(\lambda_{1}+\lambda_{\gamma}+\|\rho\|^{2}\right) s} \int_{-\infty}^{+\infty} e^{-4 \nu^{2} s}\left(\sum_{j} c_{j} \nu^{2 j}\right) e^{-2 i t_{\gamma} \gamma} d \nu
\end{aligned}
$$

where $p\left(\lambda_{o, v}\right)=\Sigma c_{j} \nu^{2 j}$. Moreover

$$
\begin{aligned}
\sum_{j} c_{j} \int_{-\infty}^{+\infty} & e^{-4 s \nu^{2} \nu^{2 j} e^{-2 i t_{\gamma} \nu} d \nu} \\
& =\sum_{j} c_{j}\left(e^{-4 s \nu^{2} \nu^{2 j}}\right)^{\wedge}\left(2 t_{\gamma}\right)=\left.\sum_{j} c_{j}^{\prime} \cdot\left(\frac{d}{d \nu}\right)^{2 j}\right|_{\nu=2 L_{\gamma}}(1 / \sqrt{s}) \exp \left(-\nu^{2} / 16 s\right) .
\end{aligned}
$$

Then there are natural numbers $c, d$ and a constant $M$ so that if $0<s<1$,

$$
\left|\left(p\left(\lambda_{\sigma, v}\right) e^{-4 \nu^{2} s}\right)^{-}\left(t_{\gamma}\right)\right|<M-t_{\gamma}^{c} s^{-d} \cdot \exp \left(-t_{\gamma}^{2} / 4 s\right)
$$


Hence, if $0<s<1$,

$$
\begin{gathered}
|\varepsilon(s)|<M^{\prime} \cdot \sum_{[\gamma] \in[\Gamma]-[e]} \operatorname{vol}\left(\Gamma_{\gamma} \backslash G_{\gamma}\right) \cdot D(\gamma)^{-1} \\
\cdot u(\gamma)^{-1} \cdot t_{\gamma}^{c} \cdot s^{-d} \cdot \exp \left(-t_{\gamma}^{2} / 4 s\right) .
\end{gathered}
$$

We want to show that $\varepsilon(s) \rightarrow 0$ exponentially, as $s \downarrow 0$. We will need to introduce more notation.

An element $\gamma \in \Gamma$ is primitive if $\gamma \neq e$ and $\gamma=\gamma_{1}^{k}$ for $\gamma_{1} \in \Gamma, k \in Z$, implies that $k= \pm 1$. It is known that if $P=\{\gamma \in \Gamma \mid \gamma$ is primitive $\}$, then (cf. De George [G])

(i) $\cup_{\gamma \in P} \cup{ }_{n=1}^{\infty}\left[\gamma^{n}\right]$ is a complete set of conjugacy class representatives.

(ii) If $\gamma$ is primitive, then $\Gamma_{\gamma^{k}}=\Gamma_{\gamma}$ is infinite cyclic generated by $\gamma$.

If $\gamma$ is primitive let $\gamma=x m_{\gamma} a_{\gamma} x^{-1}$ with $m_{\gamma} \in M, a_{\gamma} \in A^{+}$and $x \in G$. Then $\gamma^{k}=x m_{\gamma}^{k} \cdot a_{\gamma}^{k} x^{-1}$ and it is a well-known fact that $\operatorname{vol}\left(\Gamma_{\gamma^{k}} \backslash G_{\gamma^{k}}\right) \cdot u\left(\gamma^{-k}\right)=t_{\gamma}$.

On the other hand, by the proof of Lemma 7.7.11 in Wallach [Wa1]

$$
D(\gamma)^{-1}<M \cdot e^{\|\rho\|_{\gamma}} \cdot \sinh \left(t_{\gamma} / 2\right)^{m_{a}} \cdot \sinh \left(t_{\gamma}\right)^{m_{2 a}} \text {. }
$$

It is also well known that there is a lower bound $a>0$ for the sequence $\left\{t_{\gamma} \mid[\gamma] \in[\Gamma]\right\}$ (for a proof see [G, Proposition 18], for instance). Hence there exists a constant $M_{1}$ st

$$
D(\gamma)^{-1}<M, \text { for all } \gamma \in \Gamma
$$

Thus

$$
\begin{aligned}
|\varepsilon(s)| & \leqslant M^{\prime} \cdot s^{-d} \sum \operatorname{vol}\left(\Gamma_{\gamma} \backslash G_{\gamma}\right) \cdot|D(\gamma)|^{-1} u(\gamma)^{-1} t_{\gamma}^{c} \cdot \exp \left(-t_{\gamma}^{2} / 4 s\right) \\
& \leqslant M_{2} \cdot s^{-d} \exp \left(-a^{2} / 8 s\right) \cdot \sum_{[\gamma] \in[\Gamma]-[e]} t_{\gamma}^{c+1} \cdot \exp \left(-t_{\gamma}^{2} / 8 s\right) .
\end{aligned}
$$

To prove the theorem we only need to show that the series $\Sigma_{[\gamma]} t_{\gamma}^{c} \cdot \exp \left(-t_{\gamma}^{2} / 8 s\right)$ converges for all $s>0$. Set

$$
E(x)=\operatorname{card}\left\{[\gamma] \in[\Gamma] \mid t_{\gamma}<x\right\}
$$

Then, by results of De George (see [G, Corollary 2]) there exists a constant $\alpha>0$ such that

$$
E(x) \sim e^{\alpha x} / \alpha x \text { as } x \rightarrow+\infty .
$$

Hence, if $0<\varepsilon<1$, for $x$ sufficiently large

$$
(1-\varepsilon) \frac{e^{\alpha x}}{\alpha x}<E(x)<(1+\varepsilon) \frac{e^{\alpha x}}{\alpha x} .
$$

Therefore

$$
\sum_{N<t_{\gamma}<2 N} t_{\gamma}^{c} \cdot \exp \left(-t_{\gamma}^{2} / 8 s\right)<b_{N}
$$

where

$$
b_{N}=(2 N)^{c-1} \exp \left(-N^{2} / 8 s\right)\left[(1+\varepsilon) e^{2 \alpha N}-(2-2 \varepsilon) e^{\alpha N}\right] \cdot \alpha^{-1}
$$

and clearly $\Sigma_{N} b_{N}<\infty$. Q.E.D. 
We now set, for $\tau \in \hat{K}$ and $s>0$,

$$
\begin{aligned}
& \phi_{\tau}(s)=\sum_{\omega \in \hat{G}} n_{\Gamma}(\omega) \cdot\left[\tau: \omega_{\mid K}\right] e^{s\left(\lambda_{\omega}-\lambda_{r}\right)}, \\
& \psi_{\tau}(s)=\sum_{\omega \in \hat{G}} n_{\Gamma}(\omega) \cdot\left[\tau: \omega_{\mid K}\right] e^{s\left(\lambda_{\omega}-\lambda_{r}\right)} .
\end{aligned}
$$

Our next task is to relate $\phi_{\tau}(s)$ and $\psi_{\tau}(s)$.

5.2 Proposition. Let $\tau \in \hat{K}$. Then $\phi_{\tau}(s)-\psi_{\tau}(s)$ extends to an analytic function on R. (In particular it is bounded as $s \rightarrow 0^{+}$.)

Proof. Let $L^{2}(\Gamma \backslash G)=\Sigma_{\omega \in \hat{G}} n_{\Gamma}(\omega) \cdot \omega$. Set

$$
\hat{G}_{\Gamma, \tau}=\left\{\omega \in \hat{G} \mid\left[\tau: \omega_{\mid K}\right] \neq 0 \text { and } n_{\Gamma}(\omega) \neq 0\right\} .
$$

To prove the proposition it is enough to show that the set $\hat{G}_{\Gamma, \tau}-\hat{G}_{c}$ is finite. If $\omega \in \hat{G}$, by the subquotient theorem, $\omega$ imbeds in the nonunitary principal series $\pi_{\sigma, \nu}$ for some $\sigma \in \hat{M}_{\tau}$ and $\nu \in a_{\text {c }}^{*}$.

Hence $\lambda_{\omega}=\lambda_{o, \nu}=-\lambda_{\sigma}-\|\rho\|^{2}-4 \nu^{2}$ and since $\lambda_{\omega} \in \mathbf{R}$ (see [War, 4.4.4.3]) then $\nu \in \mathbf{R}$ or $\nu \in i \mathbf{R}$. If $\omega \notin \hat{G}_{c}$ then $\nu \in i \mathbf{R}$. Thus, for any $\omega \in \hat{G}_{\Gamma, \tau}-\hat{G}_{c}$,

$$
\lambda_{\omega} \geqslant-\lambda_{\sigma}-\|\rho\|^{2} \geqslant \sup \left\{\lambda_{\sigma}-\|\rho\|^{2} \mid \sigma \in \hat{M}_{\tau}\right\} \text {. }
$$

On the other hand, the series $\Sigma_{\omega} n_{\Gamma}(\omega)\left[\tau: \omega_{\mid K}\right] e^{s \lambda_{\omega}}$ converges for all $s>0$. Hence, no infinite subset of $\left\{\lambda_{\omega} \mid n_{\Gamma}(\omega) \neq 0\right\}$ can be bounded below. Q.E.D.

5.3 LEMMA. If $\phi \in C^{\infty}(G) \cap L^{1}(G)$ is such that $d_{\tau} \chi_{\tau} * \phi=d_{\tau} \phi * \chi_{\tau}=\phi$ then for all $\sigma \in \hat{M}$ the map $\nu \rightarrow \theta_{\pi, \sigma \lambda}(\phi)$ is defined and holomorphic in the open strip $S_{\rho}=\{\nu \cdot \lambda|| \operatorname{Im} \nu \mid<\|\rho\| / 2\}$ and continuous in the closed strip $\bar{S}_{\rho}$.

Proof. Since $\pi(\phi)=E_{\tau} * \pi(\phi) E_{\tau} *$, if $\left\{e_{i}\right\}_{i=1}^{n}$ is a basis of $H_{\tau^{*}}^{o, \nu \lambda}$ and $\nu \in R$,

$$
\theta_{\pi, \nu \lambda}(\phi)=\int_{G} \phi(x)\left(\sum_{i}\left\langle\pi_{\sigma, \nu \lambda}(x)\left(e_{i}\right), e_{i}\right\rangle\right) d x
$$

From Wallach [Wa1, p. 278] we see that if $g(x, \nu)=\Sigma\left\langle\pi_{\sigma, \nu \lambda}(x) e_{i}, e_{i}\right\rangle$ then, for any $U$ compact subset of $\bar{S}_{\rho}$, there is a constant $M_{U}$ so that $|g(x, \nu)|<M_{U}$ for $x \in G$, $\nu \in U$. On the other hand, the Cauchy integral formula implies that for any $z_{0} \in S_{\rho}$ there is a neighborhood $V$ and a constant $M$ so that

$$
\left|\frac{\partial}{\partial \nu} g(x, \nu)\right|<M \quad \text { for } \nu \in V, x \in G .
$$

The lemma follows by dominated convergence. Q.E.D.

We need the following result of Langlands.

5.4 THEOREM (LANGLANDS [L]). Let $G$ be a semisimple Lie group of split rank one and let $\omega \in \hat{G}$. Then one of the following is true.

(a) $\omega \in \hat{G}_{c}$.

(b) $\omega \in \hat{G}_{d}$.

(c) $\omega \in \hat{G}_{\text {comp }}$, the complementary series, that is, $\omega \simeq \pi_{\sigma, \nu \lambda}$ for some $\nu=i y$ with $0>y>-\|\rho\| / 2$. 
(d) $\omega$ is infinitesimally equivalent with $L_{\sigma, \nu}=\pi_{\sigma, \nu} / \operatorname{Ker} A(\nu)$ with $\nu=i y \lambda, 0>y>$ $-\|\rho\| / 2$, where $A(\nu): H^{o, \nu} \rightarrow H^{\sigma^{\prime},-\nu}(1 \neq s \in W(A))$ is the canonical intertwining operator (cf. Wallach [Wa1, 8.11]). Moreover $L_{\sigma, \nu}=L_{\sigma^{\prime}, \nu^{\prime}}$ iff $\sigma=\sigma^{\prime}$ and $\nu=\nu^{\prime}$.

Note. The theorem can be stated under much more general conditions but we only need the above version.

5.5 THEOREM. Let $h_{\tau, s}$ be as in 4.10 . Then $\phi_{\tau}(s)-\operatorname{vol}(\Gamma \backslash G) \cdot h_{\tau, s}(e)$ extends to a $C^{\infty}$ function on $\mathbf{R}$.

(In particular, it is bounded as $s \rightarrow 0^{+}$.)

Proof. Recall

$$
\psi_{\tau}(s)=\sum_{\omega \in \hat{\sigma}_{c}} n_{\Gamma}(\omega) \cdot\left[\tau: \omega_{\mid K}\right] \cdot e^{s\left(\lambda_{\omega}-\lambda_{r}\right)} .
$$

It is enough by 5.2 to show that the statement of the theorem holds for $\psi_{\tau}(s)-$ $\operatorname{vol}(\Gamma \backslash G) \cdot h_{\tau, s}(e)$. Let

$$
\tilde{\psi}_{\tau}(s)=\sum_{\omega \in \hat{G}_{c}} n_{\Gamma}(\omega) \cdot\left[\tau: \omega_{\mid K}\right] \cdot p\left(\lambda_{\omega}\right) e^{s\left(\lambda_{\omega}-\lambda_{)}\right)} .
$$

We first show that

$$
\tilde{\psi}_{\tau}(s)-\sum_{\omega \in \hat{G}} n_{\Gamma}(\omega) \theta_{\omega}\left(\tilde{h}_{\tau, s}\right)=u_{\tau}(s) \in C^{\infty}(\mathbf{R}) .
$$

It is enough to see that if $\omega \in \hat{G}$, then either

$$
\theta_{\omega}\left(h_{\tau, s}\right)=\left[\tau: \omega_{\mid K}\right] e^{s\left(\lambda_{\omega}-\lambda_{l}\right)} p\left(\lambda_{\omega}\right) \text { or } \theta_{\omega}\left(\tilde{h}_{\tau, s}\right)=0 .
$$

Since the first equality holds if $\omega \in \hat{G}_{c}$, by holomorphy (cf. 5.3), the same is true for $\omega \in \hat{G}_{\text {comp }}$. By the Plancherel theorem $\theta_{\omega}\left(\tilde{h}_{\tau, s}\right)=0$ for $\omega \in \hat{G}_{d}$.

Finally let $\omega=L_{o, \nu}$ be a Langlands quotient, that is, $L_{o, v}=H^{o, \nu} / \operatorname{Ker} A(v)$ where $A(\nu): H^{\sigma, \nu} \rightarrow H^{\sigma^{s},-\nu}$. If $\operatorname{Ker} A(\nu) \cap H_{\tau}^{\sigma, \nu}=0$, then $\theta_{L_{0, p}}\left(\tilde{h}_{\tau, s}\right)=\theta_{\pi_{0, p}}\left(\tilde{h}_{\tau, s}\right)$. If $\operatorname{Ker} A(\nu) \cap H_{\tau}^{\sigma, \nu} \neq 0$, then it turns out, by the definition of $p(\lambda)$, that $p\left(\lambda_{\sigma, v}\right)=0$. Thus $\pi_{\sigma, r}\left(\tilde{h}_{\tau, s}\right)=0$, by holomorphy. We now set

$$
\omega_{\tau}(s)=\sum_{\omega \in \hat{G}} n_{\Gamma}(\omega) \theta_{\omega}\left(\tilde{h}_{\tau, s}\right)-\operatorname{vol}(\Gamma \backslash G) \cdot \tilde{h}_{\tau, s}(e), \quad \text { if } s>0,
$$

and

$$
\omega_{\tau}(s)=0, \text { if } s<0 .
$$

Then, by $5.1, \omega_{\tau}(s) \in C^{\infty}(\mathbf{R})$. Hence

$$
\psi_{\tau}(s)-\operatorname{vol}(\Gamma \backslash G) \tilde{h}_{\tau, s}(e)=u_{\tau}(s)+\omega_{\tau}(s) \in C^{\infty}(\mathbf{R}) .
$$

But

$$
\begin{aligned}
\tilde{\psi}_{\tau}(s) & =e^{-s \lambda} \cdot p\left(\frac{d}{d s}\right) e^{s \lambda} \psi_{\tau}(s), \\
\tilde{h}_{\tau, s}(e) & =e^{-s \lambda} \cdot p\left(\frac{d}{d s}\right) e^{s \lambda} \cdot h_{\tau, s}(e) .
\end{aligned}
$$


Thus

$$
p\left(\frac{d}{d s}\right)\left(e^{s \lambda_{1}}\left(\psi_{\tau}(s)-\operatorname{vol}(\Gamma \backslash G) h_{\tau, s}(e)\right)\right)=e^{s \lambda_{\tau}}\left(u_{\tau}(s)+\omega_{\tau}(s)\right) .
$$

By picking $v_{\tau}(s) \in \mathcal{C}^{\infty}(\mathbf{R})$ so that $p(d / d s)\left(v_{\tau}(s)\right)=e^{s \lambda}\left(u_{\tau}(s)+\omega_{\tau}(s)\right)$ we see that

$$
\psi_{\tau}(s)-\operatorname{vol}(\Gamma \backslash G) \cdot h_{\tau, s}(e)=e^{-\lambda_{r} s}\left(v_{\tau}(s)+\sum_{j=1}^{r} q_{j}(s) e^{s \lambda_{j, \nu}}\right)
$$

where $\lambda_{\delta_{j}, v_{j}}$ are the roots of $p(\lambda)$ and $q_{j}(s)$ are polynomials of degree $N_{j}-1, N_{j}$ being the order of $\lambda_{o_{j}, v_{j}}$ as a root of $p(\lambda)$. Q.E.D.

5.6 Definition. If $f, g \in e^{\infty}\left(\mathbf{R}^{+}\right)$, then by $f \sim g$ as $s \downarrow 0$ we mean that for any natural number $n$

$$
f(s)-g(s)=O\left(s^{n}\right) \quad \text { as } s \downarrow 0 .
$$

We now state the main result of this section. The theorem is due to the author under the restriction on $\tau$ that $\left[\sigma: \tau_{\mid M}\right] \in\{0,1\}$ for all $\sigma \in \hat{M}$. Since this paper was written, the result has been generalized to arbitrary $\tau \in \hat{K}$ by De George and Wallach (see [GWa, §6]), rendering Theorem 5.5 obsolete.

5.7 TheOREM. If $\tau \in \hat{K}$ then

$$
\phi_{\tau}(s)-\operatorname{vol}(\Gamma \backslash G)\left(h_{\tau, s}(e)+\sum_{\omega \in \hat{G}_{d}} d(\omega) \cdot\left[\tau: \omega_{\mid K}\right] e^{s\left(\lambda_{\omega}-\lambda_{r}\right)}\right) \text { as } s \downarrow 0 .
$$

Proof. Let as usual $\hat{G}_{d}$ be the discrete series of $G$ and let $\hat{G}_{c}$ denote the classes corresponding to the irreducible unitary principal series or the complementary series. Let $\hat{G}_{L}=\hat{G}-\left(\hat{G}_{c} \cup \hat{G}_{d}\right)$. By $4.11(\mathrm{iv})$, we may write $h_{\tau, s}=h_{\tau, s}^{0}+h_{\tau, s}^{1}$ where $h_{\tau, s}^{1} \in \mathcal{C}^{p}(G)$ for all $p>0$. This implies that the function $\nu \rightarrow \theta_{\pi_{0, v \lambda}}\left(h_{\tau, s}^{1, s}\right)$ is entire (by 5.3 , essentially), and then

$$
\theta_{\pi_{0, \mathrm{r \lambda}}}\left(h_{\tau, s}^{1}\right)=\left[\sigma: \tau_{\mid M}\right] e^{s\left(\lambda_{0, \mathrm{r} \lambda}-\lambda_{r}\right)}
$$

for all $\nu \in \mathbf{C}$, since both sides coincide for $\nu \in \mathbf{R}$, by 4 .11(iii). This says that

$$
\theta_{\omega}\left(h_{\tau, s}^{1}\right)=\left[\tau: \omega_{\mid K}\right] e^{s\left(\lambda_{\omega}-\lambda_{r}\right)}
$$

for $\omega \in \hat{G}_{c}$. By applying 5.1 to $h_{\tau, s}^{1}$, we find that there exists $E_{\Gamma}(s) \in \mathcal{C}^{\infty}(\mathbf{R})$ such that $E_{\Gamma}(s)=0$ for $s<0$ and

$$
\operatorname{tr} \pi_{\Gamma}\left(h_{\tau, s}^{1}\right)=\operatorname{vol}(\Gamma \backslash G) h_{\tau, s}^{1}(e)+E_{\Gamma}(s) .
$$

By the Plancherel theorem

$$
h_{\tau, s}^{0}(e)=\sum_{\omega \in \hat{G}_{d}} d(\omega) \theta_{\omega}\left(h_{\tau, s}^{0}\right)
$$

and since $h_{\tau, s}^{1}=h_{\tau, s}-h_{\tau, s}^{0}$ and $\theta_{\omega}\left(h_{\tau, s}\right)=0$ if $\omega \in \hat{G}_{d}$, it follows that

$$
h_{\tau, s}^{1}(e)=h_{\tau, s}(e)+\sum_{\omega \in \hat{G}_{d}} d(\omega) \cdot \theta_{\omega}\left(h_{\tau, s}^{1}\right)
$$


On the other hand

$$
\begin{aligned}
\operatorname{tr} \pi_{\Gamma}\left(h_{\tau, s}^{1}\right) & =\sum_{\omega \in \hat{G}} n_{\Gamma}(\omega) \theta_{\omega}\left(h_{\tau, s}^{1}\right) \\
& =\phi_{\tau}(s)+\sum_{\omega \in \hat{G}-\hat{G}_{c}} n_{\Gamma}(\omega)\left(\theta_{\omega}\left(h_{\tau, s}^{1}\right)-\left[\tau: \omega_{\mid K}\right] e^{s\left(\lambda_{\omega}-\lambda_{r}\right)}\right) .
\end{aligned}
$$

By algebraic manipulations we get

$$
\begin{aligned}
h_{\tau, s}(e) & +\sum_{\omega \in \hat{G}_{d}} d(\omega)\left[\tau: \omega_{\mid K}\right] e^{s\left(\lambda_{\omega}-\lambda_{r}\right)}+\frac{1}{\operatorname{vol}(\Gamma \backslash G)} \cdot E_{\Gamma}(s) \\
= & \frac{1}{\operatorname{vol}(\Gamma \backslash G)} \phi_{\tau}(s)+\sum_{\omega \in \hat{G}_{d}}\left(\frac{n_{\Gamma}(\omega)}{\operatorname{vol}(\Gamma \backslash G)}-d(\omega)\right)\left(\theta_{\omega}\left(h_{\tau, s}^{1}\right)-\left[\tau: \omega_{\mid K}\right] e^{s\left(\lambda_{\omega}-\lambda_{r}\right)}\right) \\
& +\sum_{\omega \in \hat{G}_{L}} \frac{n_{\Gamma}(\omega)}{\operatorname{vol}(\Gamma \backslash G)}\left(\theta_{\omega}\left(h_{\tau, s}^{1}\right)-\left[\tau: \omega_{\mid K}\right] e^{s\left(\lambda_{\omega}-\lambda_{r}\right)}\right)
\end{aligned}
$$

Now let us suppose that $\tau$ is such that $\left[\sigma: \tau_{\mid M}\right] \in\{0,1\}$ for all $\sigma \in \hat{M}$. Then for any $\sigma \in \hat{M}, \nu \in \mathbf{C}, \theta_{\pi_{\sigma, \lambda}}\left(h_{\tau, s}^{1}\right)=\theta_{\omega}\left(h_{\tau, s}^{1}\right)$ where $\omega$ is the subquotient of the composition series of $\pi_{\sigma, \nu \lambda}$ that contains $\tau$ as a $K$-type. If $\omega \in \hat{G}$ and $\left[\tau: \omega_{\mid K}\right] \neq 0$ then by the subquotient theorem there exist $\sigma \in \hat{M}, \nu \in \mathbf{C}\left(\right.$ with $\left.\left[\sigma: \tau_{\mid M}\right] \neq 0\right)$ such that $\omega$ is a subquotient of $\pi_{\sigma, \nu \lambda}$. Hence under the extra assumption that $\left[\sigma: \tau_{\mid M}\right] \in\{0,1\}$ for all $\sigma \in \hat{M}$, we may conclude that

$$
\theta_{\omega}\left(h_{\tau, s}^{1}\right)=\left[\tau: \omega_{\mid K}\right] e^{s\left(\lambda_{\omega}-\lambda_{r}\right)}
$$

for any $\omega \in \hat{G}$ and $s>0$. By substituting (2) in equation (1) we prove the theorem in this case since $E_{\Gamma}(s) \sim 0$ as $s \rightarrow 0$. We observe that the difficulty for a general $\tau$ is that we cannot assert (2).

In [GWa] De George and Wallach get around this problem by using their limit formulas for multiplicities in $L^{2}(\Gamma \backslash G)$. For the sake of completeness we will sketch their proof. The starting point is equation (1). If $d_{\tau, \Gamma}(s)$ is the right-hand side of (1) set

$$
c_{\tau, \Gamma}(s)=d_{\tau, \Gamma}(s)-\frac{1}{\operatorname{vol}(\Gamma \backslash G)} \phi_{\tau}(s)
$$

By a result of Cahn-Gilkey-Wolf the asymptotic expansion of $(1 / \operatorname{vol}(\Gamma \backslash G)) \cdot \phi_{\tau}(s)$ is independent of $\Gamma$ (see [CGW]). Since $E_{\Gamma}(s) \sim 0$ as $s \rightarrow 0$, this says that $c_{\tau, \Gamma}(s)$ has an asymptotic expansion as $s \rightarrow 0$ that is independent of $\Gamma$ (here we have used the fact that $h_{\tau, s}(e)$ has an asymptotic expansion as $s \rightarrow 0$; this will be proved in $\left.\S 6\right)$.

On the other hand De George and Wallach proved that if $\omega \in \hat{G}_{d} \cup \hat{G}_{L}$ then $\theta_{\omega}\left(h_{\tau, s}^{1}\right)$ extends to an entire function of $s$. Hence, so does $c_{\tau, \mathrm{\Gamma}}(s)$. But since the Taylor expansion $c_{\tau, \Gamma}(s)$ is independent of $\Gamma$, it follows that $c_{\tau, \Gamma}(s)$ itself is independent of $\Gamma$.

Consider now a tower of subgroups of $\Gamma$, that is, a sequence $\left\{\Gamma_{j}\right\}$ such that $\Gamma_{j} \supset \Gamma_{j+1}, \Gamma_{j}$ is a normal subgroup of $\Gamma, \Gamma_{j} \backslash G$ is compact for all $j$ and $\cap_{j} \Gamma_{j}=$ $\{e\}$. By results of De George and Wallach 


$$
\begin{aligned}
& \lim _{j \rightarrow \infty} d(\omega)-\frac{n_{\Gamma_{j}}(\omega)}{\operatorname{vol}\left(\Gamma_{j} \backslash G\right)}=0 \text { if } \omega \in \hat{G}_{d} \\
& \lim _{j \rightarrow \infty} \frac{n_{\Gamma_{j}}(\omega)}{\operatorname{vol}\left(\Gamma_{j} \backslash G\right)}=0 \quad \text { if } \omega \in \hat{G}_{L} .
\end{aligned}
$$

These facts easily imply that if $\varepsilon>0$ there exists $j_{0}(\varepsilon)$ such that $\left|c_{\tau, \Gamma}(s)\right|<\varepsilon$ if $0<s<1$ and $j>j_{0}(\varepsilon)$. Since $c_{\tau, \Gamma}(s)=c_{\tau, \Gamma}(s)$ for all $j$, it follows that $c_{\tau, \Gamma}=0$. This clearly implies the result.

REMARK. It is of some interest to look at the case $\tau=1$ more closely. Theorem 5.7 asserts that $\phi_{1}(s) \sim \operatorname{vol}(\Gamma \backslash G) h_{1, s}(e)$ as $s \downarrow 0$. This could be obtained directly in this case, by observing that $\left(c_{1}(\bar{\nu} \lambda)^{*}\right)^{-1}$ and $\sigma(\nu \lambda, t)$ have no poles in the upper half plane. This fails to be true for almost any other $\tau \in \hat{K}$ and we need to make use of the decomposition granted by Theorem 4.11(iv). Thus if $\tau=1$ one picks up no residues when moving contours and $h_{1, s}\left(a_{t}\right)=\exp \left(-t^{2} / 4 s\right) \cdot O\left(t^{n}\right)$ for $t$ sufficiently large. Hence $h_{1, s}=h_{1, s}^{1} \in \mathcal{C}^{e}(G)$, for any $\varepsilon>0$. This says that $\theta_{\pi_{1, \text { d }}}\left(h_{1, s}\right)$ is entire as a function of $\nu$ and then the subquotient theorem and the classification of class-one representations (see [Ko]) imply that $\theta_{\omega}\left(h_{1, s}\right)$ has the right value for any $\omega \in \hat{G}_{1}$, that is, $\theta_{\omega}\left(h_{1, s}\right)=e^{s \lambda_{\omega}}$.

REMARK. As an example let us specialize by taking $G=\mathrm{Sl}(2, \mathrm{R})$. We will give a simple proof of 5.7 in this particular case. We have that

$$
K=\left\{K(\theta)=\left[\begin{array}{cc}
\cos \theta & \sin \theta \\
-\sin \theta & \cos \theta
\end{array}\right], \theta \in \mathbf{R}\right\} \text { and } M=\{I,-I\}
$$

Hence if $\tau_{m}(k(\theta))=e^{i m \theta}$, for $m \in Z$, then $\hat{K}=\left\{\tau_{m} \mid m \in Z\right\}$. Also $\hat{M}=\{1, \varepsilon\}$, where 1 is the trivial representation of $M$ and $\varepsilon$ is the signum representation of $M$. We now describe $\hat{G}$. Fix $k$ a natural number, let $\pi_{k}$ and $\pi_{-k}$ be the irreducible unitary representations of $G$ such that

$$
\pi_{\left.k\right|_{k}}=\sum_{\substack{m>k \\ m \equiv k+1 \bmod 2}} \tau_{m}, \quad \tau_{-k_{\mid K}}=\sum_{\substack{m<-k \\ m \equiv k+1 \bmod 2}} \tau_{m} .
$$

Also, let $\pi^{+}$and $\pi^{-}$be such that

$$
\pi_{\mid K}^{+}=\sum_{\substack{m>0 \\ m \text { odd }}} \tau_{m} \text { and } \tau_{\mid K}=\sum_{\substack{m<0 \\ m \text { odd }}} \tau_{m}
$$

and let, for $d$ a natural number, $\pi^{d}$ designate the irreducible representation of $G$ of dimension $d$. Finally, let us recall that if $\pi_{1, \nu \rho}$ and $\pi_{\varepsilon, \nu \rho}$ denote the unitary principal series of $G$, then by Frobenius reciprocity

$$
\pi_{1, \nu \rho_{\mid K}}=\sum_{\substack{m \in Z \\ m \text { even }}} \tau_{m}, \quad \pi_{\varepsilon, \nu \rho_{\mid K}}=\sum_{\substack{m \in Z \\ m \text { odd }}} \tau_{m} .
$$

We will need the following well-known classification. 
5.8 Proposition. If $G=\operatorname{Sl}(2, \mathbf{R})$ and $\pi \in \hat{G}-\{1\}$ then $\pi$ belongs to one of the following sets:

(a) $\hat{G}_{c}=\left\{\pi_{1, \nu \rho} \mid \nu \in \mathbf{R}\right\} \cup\left\{\pi_{e, \nu \rho} \mid \nu \in \mathbf{R}, \nu \neq 0\right\}$, the irreducible unitary principal series.

(b) $\hat{G}_{r}=\left\{\pi^{+}, \pi^{-}\right\}$the irreducible constituents of the reducible unitary principal series.

(c) $\hat{G}_{\text {comp }}=\left\{\pi_{1, i y \rho} \mid 0<y<1\right\}$, the complementary series.

(d) $\hat{G}_{d}=\left\{\pi_{m} \mid m \in \mathbf{Z}, m \neq 0\right\}$, the discrete series. Moreover $\pi_{m}$ is integrable if and only if $|m|>1$.

We now discuss the asymptotic expansion of $\phi_{\tau_{2 k}}(s)$, for $k \in \mathbf{Z}$. We will derive Theorem 5.7 by reduction to the spherical case. We assume that $k>0$. Indeed, it is easily seen that $\phi_{\tau_{-2 k}}(s)=\phi_{\tau_{2 k}}(s)$. By the definitions,

$$
\begin{aligned}
\phi_{\tau_{2 k}}(s) & =\sum_{\omega \in \hat{G}_{c, 1} \cup \hat{G}_{\text {comp }, ~}} n_{\Gamma}(\omega) e^{s\left(\lambda_{\omega}-\lambda_{1}\right)}+\sum_{j=1}^{k} n_{\Gamma}\left(\pi_{2 j-1}\right) e^{s\left(\lambda_{2 j-1}-\lambda_{2 k},\right.} \\
& =e^{-s \lambda_{2 k}}\left(\phi_{1}(s)+\left(n_{\Gamma}\left(\pi_{1}\right)-1\right)+\sum_{j=2}^{k} n_{\Gamma}\left(\pi_{2 j-1}\right) e^{s \lambda_{2 j-1}}\right) .
\end{aligned}
$$

Here we have used that the irreducible subquotients of the composition series of $\pi_{1, i \rho}$ are $1, \pi_{1}$ and $\pi_{-1}$. Hence $\lambda_{1}=\lambda_{\pi_{1}}=\lambda_{\pi_{-1}}=0$. On the other hand, it is well known (see for example [Wa2]) that

(i) $n_{\Gamma}\left(\pi_{ \pm 1}\right)=\operatorname{vol}(\Gamma \backslash G) d\left(\pi_{ \pm 1}\right)+1$,

(ii) $n_{\Gamma}\left(\pi_{ \pm k}\right)=\operatorname{vol}(\Gamma \backslash G) d\left(\pi_{ \pm k}\right)$ if $k>2$.

Thus

$$
\phi_{\tau_{2 k}}(s) \sim e^{-s \lambda_{2 k} \operatorname{vol}(\Gamma \backslash G)}\left(h_{1, s}(e)+\sum_{j=1}^{k} d\left(\pi_{2 j-1}\right) e^{s \lambda_{2 j-1}}\right)
$$

or

$$
\phi_{\tau_{2 k}}(s) \sim \operatorname{vol}(\Gamma \backslash G)\left(h_{\tau_{2 k, s}}(e)+\sum_{j=1}^{k} d\left(\pi_{2 j-1}\right) e^{s \lambda_{2 j-1}}\right)
$$

as $s \downarrow 0$, as was to be shown. It is of some interest to observe how in the above argument the terms in $\phi_{\tau_{2 k}}(s)$ combine to make $\phi_{\tau_{2 k}}(s) / \operatorname{vol}(\Gamma \backslash G)$ asymptotically independent of $\Gamma$ as it has to be by a result of Cahn-Gilkey-Wolf (see [CGW]). By direct inspection of the poles of $\left(c_{\tau_{2 k}}(\bar{\nu} \rho)^{*}\right)^{-1}$ in $0<\operatorname{Im}(\nu)<1$ it can be seen that $h_{\tau_{2 k}, s} \notin \mathcal{C}^{l}(G)$.

We now look at $\tau=\tau_{2 k+1}$. Then $h_{\tau_{2 k+1}, s} \in \mathcal{C}^{\prime}(G)$ since $\Gamma_{j}(i \nu-\rho)$ is holomorphic in the upper half plane for all $j$, and the poles of $\left(c_{\tau_{2 k+1}}(\bar{\nu})^{*}\right)^{-1}$ in the upper half plane are at $\nu=h i$, where $h \in \mathbf{Z}$ and $1<h<k$. This says that we may apply the Selberg trace formula to $h_{\tau_{2 k+1}, s}$. Now $\theta_{\omega}\left(h_{\tau_{2 k+1}, s}\right)=0$ if $\omega \in \hat{G}_{d}$ and by means of a holomorphy argument using 5.3 it is not hard to see that

$$
\theta_{\omega}\left(h_{\tau_{2 k+1}, s}\right)=\left[\tau_{2 k+1}: \omega_{\mid K}\right] e^{s\left(\lambda_{\omega}-\lambda_{2 k+1}\right)}
$$

for any $\omega \in \hat{G}$. The trace formula when applied to $h_{\tau_{2 k+1,5}}$ readily implies Theorem 5.7. Finally let us observe that, conversely, Theorem 5.7 implies equations (i) and (ii) above. 
6. The asymptotic expansion of $h_{\tau, s}(e)$. As we have seen in $\$ 5$, Theorem 5.7,

$$
\phi_{\tau}(s)-\operatorname{vol}(\Gamma \backslash G) h_{\tau, s}(e) \sim \omega_{\tau}(s), \quad \text { as } s \downarrow 0,
$$

where $\omega_{\tau}(s)$ is a computable entire function of $s$. Hence, the asymptotic expansion of $\phi_{\tau}(s)$ is determined by the asymptotic expansion of $h_{\tau, s}(e)$. Our final task is to derive this expansion.

Let $\mu_{\sigma}(\nu \cdot \lambda) \cdot d \nu$ be the Plancherel measure corresponding to $\sigma \in \hat{M}$ (see 4.1). If we write $\mu_{\sigma}(z)=\mu_{\sigma}(z \lambda)$ then $\mu_{\sigma}(z)$ is a meromorphic function on $C$ that restricts to an even, nonnegative, analytic function on $\mathbf{R}$ that has polynomial growth.

As usual let $\mathfrak{h}^{-} \subset \mathfrak{m}$ be maximal abelian and let $\mathfrak{h}=\mathfrak{h}^{-}+\mathfrak{a}$. Then $\mathfrak{h}_{\mathbf{c}}$ is a Cartan subalgebra of $g_{C}$. Let $\Delta$ and $\Delta_{m}$ be respectively the root systems of $\left(g_{c}, \mathfrak{h}_{C}\right)$ and $\left(\mathfrak{m}_{\mathbf{C}}, \mathfrak{h}_{\mathbf{C}}^{-}\right)$. We define an order in $\Delta$ so that positive elements on $\mathfrak{h}^{-}$are bigger than positive elements on $a$. Let finally be $($,$) the form on \mathfrak{h}^{*}$ that is dual to $B_{\mathfrak{g}} \mid \mathfrak{h} \times \mathfrak{h}$. If $\sigma \in \hat{M}$, we denote by $\lambda_{\sigma}$ its highest weight and we let $\rho_{M}=\frac{1}{2}\left(\Sigma_{\gamma \in \Delta_{m}^{+}} \gamma\right)$. Both $\lambda_{\sigma}$ and $\rho_{M}$ are trivially extended to $\mathfrak{h}_{\mathbf{C}}$. Set

$$
q_{\sigma}(z)=\prod_{\gamma \in \Delta^{+}}\left(i z(\gamma, \lambda)+\left(\gamma, \lambda_{\sigma}+\rho_{M}\right)\right) \text {. }
$$

Then (see for example [KS])

$$
\mu_{\sigma}(z)=\tilde{c} \cdot d_{\sigma} \cdot p_{\sigma}(z) \cdot \phi_{\sigma}(z)
$$

where $\tilde{c}$ is a constant depending only on $G$ (and on the choice of the Haar measure on $G), d_{\sigma}=\operatorname{dim}(\sigma)$, and $p_{\sigma}(z)$ is a monic polynomial that is a constant multiple of $q_{\sigma}(z)$. Moreover $\phi_{\sigma}(z)=1$, if $g \simeq \operatorname{SO}(2 n+1,1)$, and otherwise $\phi_{\sigma}(z)=\tanh \pi z$ or coth $\pi z$, depending on $\sigma$. The choice of tanh or coth is done roughly as follows. There is a distinguished element $\beta \in \exp \mathfrak{h}^{-}$of order at most 2. If $H \in \mathfrak{h}^{-}$ satisfies $\exp (H)=\beta$, then $\exp \left(\lambda_{\sigma}+\rho_{M}\right)(H)= \pm 1$. The coth is used when the sign is + and the tanh when the sign is - . We have computed the polynomial $p_{\sigma}(z)$ and applied the above criterion to linear rank one groups to get a very explicit expression of the Plancherel measure (see [M]).

From the definition of $h_{\tau, s}$ we see that

$$
h_{\tau, s}(e)=e^{-\left(\lambda_{+}+\|\rho\|^{2}\right) s} \cdot \sum_{\sigma \in \hat{M}_{\tau}} e^{-s \lambda_{\sigma} a} \cdot\left[\sigma: \tau_{\mid M}\right] \int_{-\infty}^{+\infty} e^{-4 s z^{2}} \cdot \mu_{\sigma}(z) d z
$$

Let us set

$$
\begin{aligned}
& \hat{M}_{\tau, t}=\left\{\sigma \in \hat{M} \mid\left[\sigma: \tau_{\mid M}\right] \neq 0 \text { and } \mu_{\sigma}(z) \text { goes with tanh }\right\}, \\
& \hat{M}_{\tau, c}=\left\{\sigma \in \hat{M} \mid\left[\sigma: \tau_{\mid M}\right] \neq 0 \text { and } \mu_{\sigma}(z) \text { goes with coth }\right\} .
\end{aligned}
$$

We first assume that $g \approx \operatorname{SO}(2 n+1,1)$. Then $\hat{M}_{\tau}=\hat{M}_{\tau, t} \cup \hat{M}_{\tau, c}$ and $p_{\sigma}(z)$ is odd. Let us write $p_{\sigma}(z)=\sum_{i=0}^{l} b_{2 i+1}(\sigma) \cdot z^{2 i+1}$ and $c=\frac{1}{2} \cdot \operatorname{dim}(G / K)$. Then $c=l$ +1 .

Let us set

$$
\begin{aligned}
h_{\tau, s}(e)_{t}= & e^{-\left(\lambda_{t}+\|\rho\|^{2}\right) s} \cdot \sum_{\sigma \in \hat{M}_{\tau, t}} \tilde{c} \cdot d_{\sigma} \cdot\left[\sigma: \tau_{\mid M}\right] \cdot e^{s \lambda_{,} a} \\
& \cdot \sum_{i=0}^{l} b_{2 i+1}(\sigma) \cdot \int_{-\infty}^{+\infty} z^{2 i+1} \cdot e^{-4 s z^{2}} \cdot \tanh \pi z d z
\end{aligned}
$$


We define $h_{\tau, s}(e)_{c}$ similarly, by using $\hat{M}_{\tau, c}$ and coth $\pi z$. We have that $h_{\tau, s}(e)=$ $h_{\tau, s}(e)_{t}+h_{\tau, s}(e)_{c}$. To obtain the asymptotic expansion of $h_{\tau, s}(e)$ we need some facts from Fourier transform theory. Let $S(R)$ denote the Schwartz space of $\mathbf{R}$ and $S^{\prime}(\mathbf{R})$ the space of tempered distributions. If $f \in S(\mathbf{R}), T \in S^{\prime}(\mathbf{R})$ let

$$
\begin{aligned}
\hat{f}(x) & =\int_{-\infty}^{+\infty} f(t) \cdot e^{-i t x} d t, \\
f^{\nu}(x) & =\int_{-\infty}^{+\infty} f(t) \cdot e^{i t x} d t,\left\langle T^{\wedge}, f\right\rangle=\langle T, \hat{f}\rangle,\left\langle T^{-}, f\right\rangle=\left\langle T, f^{-}\right\rangle .
\end{aligned}
$$

6.1 Proposition. (i) If $T \in S^{\prime}(\mathbf{R}),\left(T^{\prime}\right)^{\nu}=\left(T^{\prime}\right)^{\wedge}=2 \pi \cdot T$.

(ii) If $a>0$ and $f(t)=e^{-a t^{2}}$, then

$$
\hat{f}(x)=f^{\nu}(x)=\sqrt{\pi / a} \cdot \exp \left(-x^{2} / 4 a\right) .
$$

(iii) Let $h(t)=\tanh \pi t \in S^{\prime}(\mathbf{R})$. Then

$$
h^{\wedge}=-i \cdot p \cdot v \operatorname{cosech}(t / 2) \text {. }
$$

If $T=p \cdot v \operatorname{coth} \pi t$, then

$$
T^{\wedge}=-i \cdot p \cdot v \operatorname{coth}(t / 2) .
$$

Therefore, 6.1 implies that

$$
\begin{aligned}
\int z^{2 i+1} \cdot e^{-4 s z^{2}} \cdot \tanh \pi z d z=(2 \pi)^{-1}\left\langle\tanh \pi x,\left(z^{2 i+1} \cdot e^{-4 s z^{2}}\right)^{-a}\right\rangle \\
=(-1)^{i+1} \cdot(\sqrt{16 \pi s})^{-1} \cdot\left\langle p \cdot v \operatorname{cosech}(x / 2), D^{2 i+1} \exp \left(-x^{2} / 16 s\right)\right\rangle .
\end{aligned}
$$

(Here $D=d / d x$.) If $H_{2 i+1}(x)$ is the Hermite polynomial of order $2 i+1$ then

$$
H_{2 i+1}(x)=\sum_{h=0}^{i} c_{h, 2 i+1} \cdot x^{2 h+1}
$$

where

$$
c_{h, 2 i+1}=\frac{(-1)^{i-h+1} \cdot(2 i+1) ! 2^{2 h+1}}{(i-h) !(2 h+1) !} .
$$

Moreover

$$
D^{2 i+1} \exp \left(-a x^{2}\right)=\left(\sum_{h=0}^{i} a^{h+i+1} \cdot c_{h, 2 i+1} \cdot x^{2 h+1}\right) \exp \left(-a x^{2}\right) .
$$

Hence, the above can be written

$$
\begin{aligned}
= & (-1)^{i+1} \cdot(\sqrt{\pi s})^{-1} \cdot \sum_{h=0}^{i} 2^{-2 h-4 i-4} \cdot s^{-h-i-1} \\
& c_{h, 2 i+1}\left\langle p \cdot v \operatorname{cosech} x, x^{2 h+1} \exp \left(-x^{2} / 4 s\right)\right\rangle .
\end{aligned}
$$

Let $x \operatorname{cosech} x=\Sigma_{j} d_{j} x^{2 j}$, for $|x|<\pi$. Using that $\int_{-\infty}^{+\infty} x^{2 k} \cdot e^{-x^{2}} d x=\Gamma\left(k+\frac{1}{2}\right)$ it is not hard to show that $\left\langle p \cdot v \operatorname{cosech} x, x^{2 h+1} \cdot \exp \left(-x^{2} / 4 s\right)\right\rangle$ is asymptotic in the sense of 5.6 to $\Sigma_{0}^{\infty} d_{j} \cdot \Gamma\left(h+j+\frac{1}{2}\right) \cdot(\sqrt{4 s})^{2 h+2 j+1}$, as $s \rightarrow 0^{+}$. 
Hence, adding over $i$ and letting $m=c-i-1, n=c+j-i-1$, it follows that

$$
\begin{aligned}
& \sum_{i=0}^{l} b_{2 i+1}(\sigma) \int_{-\infty}^{+\infty} z^{2 i+1} \cdot e^{-4 s z^{2}} \cdot \tanh \pi z d z \\
& \sim s^{-c}\left(\sum_{n=0}^{\infty} \sum_{h=0}^{c-1-v} \sum_{v=0}^{\max (c-1, n)}(\sqrt{\pi})^{-1}(-1)^{c-v} \cdot 2^{2 n-4 c+2 v+1}\right. \\
&\left.\cdot b_{2 c-2 v-1}(\sigma) \cdot d_{n-v} \cdot c_{h, 2 c-2 v-1} \cdot s^{n}\right) .
\end{aligned}
$$

Hence, as $s \rightarrow 0^{+}$,

$$
h_{\tau, s}(e)_{t} \sim e^{-\left(\lambda_{t}+\|\rho\|^{2}\right) s} \cdot s^{-c}\left(\sum_{n=0}^{\infty} a_{n, t}(s) \cdot s^{n}\right),
$$

where

$$
\begin{aligned}
a_{n, t}(s)= & \sum_{\sigma \in \hat{M}_{\tau, t}} e^{-s \lambda_{0} \cdot a} \cdot\left[\sigma: \tau_{\mid M}\right] \sum_{h=0}^{c-1-v} \sum_{v=0}^{\max (c-1, n)}(-1)^{c-v} \cdot(\sqrt{\pi})^{-1} \\
& \cdot 2^{2 n-4 c+2 v+1} \cdot b_{2 c-2 v-1}(\sigma) \cdot d_{n-v} \cdot c_{h, 2 c-2 v-1} \cdot \Gamma\left(h+n-v+\frac{1}{2}\right) .
\end{aligned}
$$

To derive the expansion for $h_{\tau, s}(e)_{c}$ we proceed similarly and arrive at

$$
h_{\tau, s}(e)_{c} \sim e^{-\left(\lambda+\|\rho\|^{2}\right) s} \cdot s^{-c}\left(\sum_{n=0}^{\infty} a_{n, c}(s) \cdot s^{n}\right), \quad \text { as } s \rightarrow 0^{+} .
$$

Here $a_{n, c}(s)$ is given by the same formula as $a_{n, t}(s)$ except that $d_{j}$ is replaced by $d_{j}^{\prime}$, the coefficients of the Taylor expansion at $z=0$ of $z \cdot \operatorname{coth} z$.

When $G$ is locally isomorphic to $\operatorname{SO}(2 n+1,1)$, the Plancherel measure is a polynomial: $\mu_{\sigma}(z)=\sum_{i=0}^{l} b_{2 i}(\sigma) \cdot z^{2 i}$ and $l=c-\frac{1}{2}$. Thus

$$
\begin{aligned}
h_{\tau, s}(e) & =e^{-\left(\lambda_{r}+\|\rho\|^{2}\right) s} \cdot \sum_{\sigma \in \hat{M}_{\tau}}\left[\sigma: \tau_{\mid M}\right] e^{-s \lambda_{j} \cdot a} \cdot \sum_{i=0}^{l} b_{2 i}(\sigma) \cdot \Gamma\left(i+\frac{1}{2}\right) \\
& =e^{-\left(\lambda_{r}+\|\rho\|^{2}\right) s} \cdot s^{-c}\left(\sum_{h=0}^{l} \sum_{\sigma \in \hat{M}_{\tau}}\left[\sigma: \tau_{\mid M}\right] \cdot e^{-s \lambda_{j} \cdot a} \cdot b_{2 c-2 h-1}(\sigma) \cdot \Gamma(c-h) \cdot s^{h}\right) .
\end{aligned}
$$

Summing up:

6.2 ThEOREM. For $\tau \in \hat{K}, s>0$, let $h_{\tau, s}$ be as in 4.10. Then, as $s \rightarrow 0^{+}$,

$$
h_{\tau, s}(e) \sim s^{-c} \cdot e^{-s\left(\lambda+\|\rho\|^{2}\right)} \cdot\left(\sum_{j=0}^{\infty} a_{j}(s) s^{j}\right)
$$

where $c=\frac{1}{2} \operatorname{dim}(G / K)$ and the coefficients $a_{j}(s)$ are as follows 
(i) If $\mathfrak{g} \approx \mathrm{SO}(2 n+1,1)$, then

$$
\begin{aligned}
& a_{j}(s)=\sum_{h=0}^{c-1-v} \sum_{v=0}^{\max (c-1, j)}(-1)^{c-v} \cdot(\sqrt{\pi})^{-1} \cdot c_{h, 2 c-2 v-1} \cdot 2^{2 n-4 c+2 v+1} \\
& \cdot \Gamma\left(h+n-v+\frac{1}{2}\right)\left[\sum_{\sigma \in \hat{M}_{\tau, c}} d_{n-v} \cdot\left[\sigma: \tau_{\mid M}\right] \cdot b_{2 c-2 v-1}(\sigma) \cdot e^{-s \lambda_{j} \cdot a}\right. \\
&\left.+\sum_{\sigma \in \hat{M}_{\tau, c}} d_{n-v}^{\prime} \cdot\left[\sigma: \tau_{\mid M}\right] \cdot b_{2 c-2 v-1}(\sigma) \cdot e^{-s \lambda_{r} \cdot a}\right] .
\end{aligned}
$$

(ii) If $\mathfrak{g} \simeq \mathrm{SO}(2 n+1,1)$

$$
a_{j}(s)=\sum_{\sigma \in \hat{M}_{\tau}}\left[\sigma: \tau_{\mid M}\right] e^{-s \lambda_{0} \cdot a} \cdot b_{2 c-2 j-1}(\sigma) \cdot \Gamma(c-j)
$$

if $j=0,1, \ldots,(2 c-1) / 2$ and $a_{j}(s)=0$, if $j>(2 c-1) / 2$.

Let us recall that

$$
c_{h, 2 i+1}=\frac{(-1)^{i-h+1}(2 i+1) ! \cdot 2^{2 h+1}}{(i-h) !(2 h+1) !}
$$

and if $j \neq 0$

$$
d_{j}=\frac{(-1)^{j}\left(2^{2 j}-2\right) B_{j}}{2 j !}, \quad d_{j}^{\prime}=\frac{(-1)^{j-1} \cdot B_{j} \cdot 2^{2 j}}{2 j !}
$$

Moreover $d_{0}=d_{0}^{\prime}=1$, and $a=B_{\mathrm{g}}\left(H_{1}, H_{1}\right)$.

If $q_{\sigma}(z)$ denotes the polynomial part of the Plancherel measure, then $q_{\sigma}(z)=$ $\Sigma_{j=0}^{2 c-1} b_{j}(\sigma) \cdot z^{j}$.

Remark. We observe that when $\tau=1$, the functions $a_{j}(s)$ are constants. In general there is an asymptotic expansion $h_{\tau, s}(e) \sim s^{-c}\left(\sum_{j=0}^{\infty} c_{j} \cdot s^{j}\right), c_{j} \in \mathbf{R}$, as $s \rightarrow$ $0^{+}$, and the $c_{j}$ 's are determined by expanding the $a_{j}(s)$ in Taylor series and proceeding formally in the obvious manner.

\section{REFERENCES}

[ABP] M. Atiyah, R. Bott and V. K. Patodi, On the heat equation and the index theorem, Invent. Math. 19 (1973), 279-330.

[BGM] M. Berger, P. Gauduchon and E. Mazet, Le spectre d' une variété Riemannienne, Lecture Notes in Math., vol. 194, Springer-Verlag, Berlin and New York, 1971.

[CW] R. Cahn and J. Wolf, Zeta functions and their asymptotic expansions for compact symmetric spaces of rank one, Comment. Math. Helv. 51 (1976), 1-21.

[CGW] R. Cahn, P. Gilkey and J. Wolf, Heat equation, proportionality principle, and volume of fundamental domains, Math. Phys. Appl. Math., vol. 3, Reidel, Dordrecht, 1976, pp. 43-54.

[C] O. Campoli, The complex Fourier transform on rank one semisimple Lie groups, Thesis, Rutgers University, 1976.

[G] D. De George, Length spectrum for compact locally symmetric spaces of strictly negative curvature, Ann. Ecole Norm. Sup. 10 (1977), 133-152.

[GWa] D. De George and N. Wallach, Limit formulas for multiplicites in $L^{2}(\Gamma \backslash G)$. II. The tempered spectrum, Ann. of Math. (to appear).

[Ga] R. Gangolli, Asymptotic behaviour of spectra of compact quotients of certain symmetric spaces, Acta Math. 121 (1968), 151-192. 
[GW] R. Gangolli and G. Warner, On Selberg's trace formula, J. Math. Soc. Japan 27 (1975), 328-343.

[GGP] I. M. Gel'fand, M. Graev and I. Pyatetskii-Shapiro, Representation theory and automorphic functions, Saunders, Philadelphia, Pa., 1969.

[HC1] Harish-Chandra, Discrete series for semisimple Lie groups. II, Acta Math. 116 (1966), 1-111.

[HC2] , Harmonic analysis on real reductive groups. III. The Maass-Selberg relations and the Plancherel formula, Ann. of Math. (2) 104 (1976), 117-201.

[HS] H. Hecht and W. Schmid, A proof of Blattner's conjecture, Invent. Math. 31 (1975), 129-154.

[He] S. Helgason, A duality for symmetric spaces with applications to group representations, Advances in Math. 5 (1970), 1-154.

[J] K. Johnson, Differential equations and an analog of the Paley Wiener theorem for linear semisimple Lie groups, Nagoya Math. J. 64 (1976), 17-29.

[KS] A. Knapp and E. Stein, Intertwining operators for semisimple groups, Ann. of Math. (2) 93 (1971), 489-578.

[Ko] B. Kostant, On the existence and irreducibility of certain series of representations, Bull. Amer. Math. Soc. 75 (1969), 627-642.

[L] R. Langlands, On the classification of irreducible representations of algebraic groups, Institute for Advanced Study, Princeton, N.J., 1975 (preprint).

[MS] H. McKean and I. Singer, Curvature and the eigenvalues of the Laplacian, J. Differential Geometry 1 (1967), 43-69.

[M] R. Miatello, On the Plancherel measure for linear Lie groups of rank one, Manuscripta Math. 29 (1979), 249-276.

[MP] S. Minakshisundaram and A. Pleijel, Some properties of the eigenfunctions of the Laplace operator on Riemannian manifolds, Canad. J. Math. 1 (1949), 242-256.

[P] R. Palais et al., Seminar on the Atiyah-Singer index theorem, Ann. of Math. Studies, vol. 59, Princeton Univ. Press, Princeton, N.J., 1965.

[R] M. Raghunathan, Discrete subgroups of Lie groups, Springer-Verlag, Berlin and New York, 1972.

[Wa1] N. R. Wallach, Harmonic analysis on homogeneous spaces, Dekker, New York, 1973.

[Wa2] _ On the Selberg trace formula in the case of compact quotient, Bull. Amer. Math. Soc. 82 (1976), 171-195.

[W/2] _ On an asymptotic formula of Gelfand and Gangolli for the spectrum of $\Gamma \backslash G, \mathrm{~J}$. Differential Geometry 11 (1976), 91-101.

[Wa4] __, On Harish-Chandra's generalized c-functions, Amer. J. Math. 97 (1975), 386-403.

[War] G. Warner, Harmonic analysis on semisimple Lie groups. I, Springer-Verlag, Berlin and New York, 1972.

Departamento de Matemítica, Universidade federal de Pernambuco, 50.000 Recife, PerNAMBUCO, BRAZIL 